\title{
Numerical studies on issues of Re-independence for indoor airflow and pollutant dispersion within an isolated building
}

\author{
Peng-Yi Cui', Wei-Qiu Chen', Jia-Qi Wang' ${ }^{1}$ Jin-Hao Zhang' ${ }^{1}$ Yuan-Dong Huang ${ }^{1}(\varangle)$, Wen-Quan Tao \\ 1. College of Environment and Architecture, University of Shanghai for Science and Technology, Shanghai, China \\ 2. Key Laboratory of Thermo-Fluid Science and Engineering of MOE, School of Energy and Power Engineering, Xi'an Jiaotong University, \\ Xi'an, China
}

\begin{abstract}
This study conducted the numerical models validated by wind-tunnel experiments to investigate the issues of Re-independence of indoor airflow and pollutant dispersion within an isolated building. The window Reynolds number $\left(R e_{w}\right)$ was specified to characterize the indoor flow and dispersion. The indicators of $R R C$ (ratio of relative change) or $D R\left(K \_D R\right)$ (difference ratio of dimensionless concentration) $\leq 5 \%$ were applied to quantitatively determine the critical $R e_{w}$ for indoor flow and turbulent diffusion. The results show that the critical $R e$ ( $R e_{\text {crit }}$ ) value is position-dependent, and $R e_{\text {crit }}$ at the most unfavorable position should be suggested as the optimal value within the whole areas of interest. Thus $R_{\mathrm{H}, \text { crit }}=27,000$ is recommended for the outdoor flows; while $R e_{w, \text { crit }}=15,000$ is determined for the indoor flows due to the lower part below the window showing the most unfavorable. The suggested $R_{w, \text { crit }}(=15,000)$ for indoor airflow and cross ventilation is independence of the window size. Moreover, taking $K \_D R \leq 5 \%$ as the indicator, the suggested $R e_{w, \text { rrit }}$ for ensuring indoor pollutant diffusion enter the $R e$-independence regime should also be 15,000 , indicating that indoor passive diffusion is completely determined by the flow structures. The contours of dimensionless velocity $\left(U / U_{0}\right)$ and concentration $(K)$ against the increasing $R e_{w}$ further confirmed this critical value. This study further reveals the Re-independence issues for indoor flow and dispersion to ensure the reliability of the data obtained by reduced-scale numerical or wind-tunnel models.
\end{abstract}

\section{Keywords}

CFD simulation;

Re-independence;

similarity criterion;

indoor ventilation;

pollutant dispersion

\section{Article History}

Received: 23 May 2021

Revised: 17 August 2021

Accepted: 07 September 2021

(C) Tsinghua University Press and Springer-Verlag GmbH Germany, part of Springer Nature 2021

\section{Introduction}

In recent decades, with the rapid development of economy and the continuous improvement of people's living standards, the requirements for good life quality, living comfort and safety are increasing. Previous studies show that most people spend more than $85 \%$ of their times in indoor environment, therefore, indoor air quality has a direct impact on human health (Robinson and Nelson 1995). In addition to partial sources generated from decoration materials and daily life (smoking, cooking, etc.), most of the indoor air pollutants are from outdoor atmospheric environment due to ventilation, such as industrial and traffic emissions and more complex secondary pollutants (such as haze or photochemical smog (Jones 1999)). Besides, respiratory viruses such as novel
Coronavirus (COVID-19) may be transmitted in the air by sticking to the droplets or aerosols, and thus may enter the room through ventilation from the outside or other building floors (Jayaweera et al. 2020; Wilson et al. 2020). Researches show that natural ventilation is the most economical and effective way to remove indoor air pollutants, so good ventilation design is particularly important to improve indoor environment (Jiru and Bitsuamlak 2010).

The on-site full-scale experiment, reduced-scale wind-tunnel modeling and numerical simulation (CFD, computational fluid dynamics) are main approaches used to study indoor natural ventilation and pollutant dispersion. Although the on-site full-scale experiment can better reflect the real situation, unfavorable factors such as huge workload, high cost and unpredictable meteorological conditions greatly

E-mail: huangyd@usst.edu.cn 
limit its further development. In consideration of being likely inhibited by $R e$ similarity criterion near the narrow ventilation openings for the reduced-scale wind-tunnel experiments, van Hooff and Blocken (2010a) performed an on-site full-scale measurement to obtain the parametric analysis of natural ventilation in a complex semi-enclosed stadium, such as air exchange rate $(\mathrm{ACH})$ based on $\mathrm{CO}_{2}$ decay. Then these measured results were used to provide validation data for the established numerical model coupled urban wind flow and indoor natural ventilation (van Hooff and Blocken 2010b). The on-site full-scale experiment was conducted by Omrani et al. (2017) to measure the effect of natural ventilation modes (single-sided and cross ventilation) on indoor thermal comfort and ventilation performance. The measured data such as wind speed, air temperature and relative humidity coupled with two comprehensive parameters, PMV (predicted mean vote) and SET ${ }^{\star}$ (standard effective temperature) were used to evaluate the indoor thermal environment. A numerical model of an isolated building with a wind catcher incorporated heat pipes was established by Calautit and Hughes (2016) to study the cooling performance of the wind catcher. Due to the limitation of similarity criterion, the reduced-scale wind-tunnel tests were only used to validate the flow full-scale without considering the thermal effect, while the thermal performance under real operating conditions had to be evaluate by the on-site tests.

Wind-tunnel experiments with a controllable inflow conditions can be applied to simulate the different types of atmospheric boundary layer profiles. However, during windtunnel experiments, reduced-scale building models are often required (scale ratio varies from 1:5 to $1: 300$ ), therefore, certain similarity criteria must be followed to ensure the reliability of the wind-tunnel tests (Townsend 1956; Snyder 1981). CFD technology developed in recent years is an important means to study airflow, heat transfer and pollutant dispersion within different scale urban areas. Compared to on-site full-scale experiment and wind-tunnel test, it has obvious advantages such as low cost, controllable boundary conditions, and rich data acquisition, etc. However, the experimental data is needed to ensure the accuracy and reliability of the numerical models. In previous studies, RANS and LES are two common turbulent models to be applied to simulate the urban flow and pollutant dispersion, and the model validation often proves that LES has higher numerical accuracy (Gousseau et al. 2011; Ramponi and Blocken 2012; Gilani et al. 2016; Shirzadi et al. 2018).

In addition, to save computational resource, the reducedscale numerical models are often applied to study the physical phenomena of airflow, heat and mass transfer. As we known, the prediction accuracy of numerical simulations depends heavily on the appropriateness of grid resolution. Particularly, the high-density grid resolution is required for the accuracy predictions of near-field complex environmental processes, such as the separating flows around building obstacles, which cannot be correctly reproduced based on a coarse grid. And in fact, the near-wall $z+$ values, namely the nondimensional distance between the first cell centroid and its closet wall, are specifically required by specific turbulence models and near-wall treatment approaches (Franke et al. 2007; Tominaga et al. 2008; Tominaga and Stathopoulos 2013; Ai and Mak 2014a). Ai and Mak (2014a) proposed this hypothesis that reduced-scale numerical models can largely reduce computing consumption, and the theoretical analysis and numerical studies coupled with wind-tunnel tests to verify this hypothesis. And they found that the reduced-scale numerical model requires much less grid numbers to achieve a target near-wall $z+$ value and higher precision numerical results than that of full-scale numerical simulation, but the similarity criterion needs to be satisfied firstly. Given this, Gromke (2011) conducted wind-tunnel experiments to study the impact of tree planting on airflow and vehicle exhaust diffusion within street canyons, where porous materials were used to simulate the aerodynamic impact of tree planting in street canyons and the similar criteria followed by wind-tunnel experiments were introduced in details. Then a reduced-scale numerical model of a street canyon with tree planting was built, and the accuracy of two turbulence models (standard $k-\varepsilon$ model, SKE and Reynolds stress model, RSM) was evaluated, showing that the RSM model performed much better (Gromke et al. 2008). Moreover, the reduced-scale numerical model with RSM model was further applied to study the effects of the width-to-height ratios of the street canyon, crown porosity, tree planting density, wind directions, tree planting pattern and trunk height on the airflow and pollutant dispersion inside a street canyon (Gromke and Ruck 2012; Huang et al. 2019). Similar with the reduced-scale wind-tunnel experiment, the reduced-scale numerical model should also consider the relative similarity criteria to ensure that the model results can reasonably reflect the real world. However, a considerable number of previous numerical studies with reduced-scale model on indoor ventilation, heat transfer and pollutant dispersion has not clearly stated the relative similar criteria (Chow et al. 2014; Cui et al. 2014, 2017; Park et al. 2017; van Hooff et al. 2017; Shirzadi et al. 2020).

According to Snyder (1972), the equations describing the phenomena of flow and dispersion are normalized to indicate that five dimensionless numbers, namely Reynolds $(R e)$, Froude $(F r)$, Rossby $(R o)$, Peclet $(P e)$, and Schmidt $(S c)$ numbers, should be satisfied. However, due to the complexity of actual environmental conditions, it is impractical and impossible to satisfy all the similar criteria simultaneously. Therefore, it is an important principle for reduced-scale model design to satisfy the primary similarity criterion and 
ignore the less important criterion. Snyder (1972, 1981) pointed out that when modeling prototype flows with a length scale less than about $5 \mathrm{~km}$ the Ro number can be neglected. And the $R e, P e$ and $S c$ number criteria may be neglected if the model flow is of sufficiently high $R e$. Then in such a case, $F r$ number (driven by density difference) seems to be the only one which should be matched between the prototype and the reduced-scale wind-tunnel test. However, the Re number is still abused in modeling flows inside or around buildings, because the question: how large must the Re number be to be "sufficiently high" or "large enough"? should be solved (Cui et al. 2014). Under the urban boundary layer, $R e$ (flow similarity) and $\mathrm{Fr}$ (buoyancy effect) are two most important similarity criteria for investigating the indoor and outdoor ventilation and pollutant dispersion with reduced-scale models. Generally, the criterion of the equivalent $R e$ between the model and the prototype is difficult to satisfy. Fortunately, the proposed principle of $R e$-independence makes this problem solved effectively, that is, when $R e$ is greater than a critical value, the flow structure over a rough surface would be almost independent on the Reynolds number. Therefore, it is of great significance to obtain the critical $R e\left(R e_{\text {crit }}\right)$ to ensure the $R e$-independence of flow structures in the region concerned.

Previous studies on Re-independent problems mainly focused on the external flow around the buildings and mainly adopted the building height Reynolds number, $R e_{\mathrm{H}}$ and the roughness Reynolds number, $R e_{\mathrm{r}}$. Table 1 lists the critical $R e_{\mathrm{H}}\left(R e_{\mathrm{H}, \text { crit }}\right)$ and the critical $R e_{\mathrm{r}}\left(R e_{\mathrm{r}, \text { ritit }}\right)$ adopted by previous studies on the external flow around buildings. Among them, the ranges of $R e_{\mathrm{H}, \text { crit }}$ from thousands to tens of thousands, mainly due to the different characteristic height of building adopted. Nevertheless, perhaps due to the complexity of the calculations, $R e_{\text {r,crit }}$ ranging from a few to a dozen are rarely adopted in literatures. However, since the criterion of $R e$-independence has been put forward, there are only a little literature specifically to study the critical Re. Uehara et al. (2003) conducted wind-tunnel experiments to compare the changes of flow structures around the building models, and qualitatively suggested the critical values of $R e_{\mathrm{H}}(3,500-8,000)$ and $R e_{\mathrm{r}}$ (5.4). In our previous work, a quantitative standard of $R R C$ (ratio of relative change) $\leq 5 \%$ was firstly proposed to quantitatively determine the critical values of $R e_{\mathrm{H}}\left(3.3 \times 10^{4}\right)$ and $R e_{\mathrm{r}}(5.4)$ (Cui et al. 2014, 2017).

Obviously, the Re-independence for indoor airflow and pollutant dispersion is more difficult to satisfy because air flowing in a narrow space is subject to greater viscous forces. In previous studies by using reduced-scale wind-tunnel or numerical models, the issue of $R e$-independence for the indoor airflow and pollutant dispersion has not attracted much attention. Instead, the critical Re for the outdoor airflow around buildings was still adopted (Ikegaya et al. 2019; Kosutova et al. 2019; Shirzadi et al. 2020). Dai et al. (2019) conducted numerical studies on the issue of Reindependence of airflow and pollutant dispersion by coupling the indoor and outdoor environment. They suggested that the critical $R e_{\mathrm{H}}$ and $R e_{\mathrm{w}}$ for the outdoor and indoor flow structures are $4.8 \times 10^{4}$ and $1.4 \times 10^{4}$, respectively, while the critical values for pollutant dispersion are $7.9 \times 10^{4}$ and $3.0 \times 10^{4}$, respectively. The Re-independence of airflow and pollutant dispersion within an underground sewage treatment

Table 1 The critical Reynolds numbers adopted by previous studies on the external flow around buildings

\begin{tabular}{|c|c|c|}
\hline Reference & The type of Reynolds number & The value of $R e_{\text {crit }}$ \\
\hline \multirow{2}{*}{ Snyder 1972, 1981} & $R e_{\mathrm{H}}$ (building Reynolds number) & $4.0 \times 10^{3}$ \\
\hline & $R e_{\mathrm{r}}$ (roughness Reynolds number) & 2.5 \\
\hline Castro and Robins 1977 & $R e_{\mathrm{H}}$ (building Reynolds number) & $4.0 \times 10^{3}$ \\
\hline Cherry et al. 1984 & $R e_{\mathrm{H}}$ (building Reynolds number) & $3.0 \times 10^{4}$ \\
\hline Meroney 1987 & $R e_{\mathrm{H}}$ (building Reynolds number) & $1.1 \times 10^{4}-1.0 \times 10^{5}$ \\
\hline Ohba 1989 & $R e_{\mathrm{H}}$ (building Reynolds number) & $2.1 \times 10^{3}$ \\
\hline Djilali and Gartshore 1991 & $R e_{\mathrm{H}}$ (building Reynolds number) & $2.5 \times 10^{4}$ \\
\hline Mochida et al. 1994 & $R e_{\mathrm{H}}$ (building Reynolds number) & $7.5 \times 10^{3}$ \\
\hline Saathoff et al. 1995, 2009 & $R e_{\mathrm{b}}$ (building Reynolds number) & $1.1 \times 10^{4}$ \\
\hline \multirow{2}{*}{ Uehara et al. 2003} & $R e_{\mathrm{H}}$ (building Reynolds number) & $3.5 \times 10^{3}-8.0 \times 10^{3}$ \\
\hline & $R e_{\mathrm{r}}$ (roughness Reynolds number) & 5.4 \\
\hline Yee et al. 2006 & $R e_{\mathrm{H}}$ (building Reynolds number) & $4.0 \times 10^{3}$ \\
\hline Lim et al. 2007 & $R e_{\mathrm{H}}$ (building Reynolds number) & $2.0 \times 10^{4}-3.0 \times 10^{4}$ \\
\hline Gupta et al. 2012 & $R e_{\mathrm{b}}$ (building Reynolds number) & $1.1 \times 10^{4}$ \\
\hline \multirow{2}{*}{ Cui et al. 2014, 2017} & $R e_{\mathrm{H}}$ (building Reynolds number) & $3.4 \times 10^{4}$ \\
\hline & $R e_{\mathrm{r}}$ (roughness Reynolds number) & 25 \\
\hline
\end{tabular}


plant was numerically investigated by a 1:40 scaled model, and two evaluation indexes (RERV and $D R$ ) were used to determine the critical $R e\left(R e_{\text {crit }}=6,000\right)$ (Dong et al. 2019). Even so, the issues on $R e$-independence of indoor flow structures and pollutant dispersion still need to be further examined, such as the effects of the open-window dimension, ventilation mode, surrounding buildings, etc. This study will further continue to focus on the solution of this problem.

The rest of this study is organized as follows. Physical model and numerical model (governing equations, grid generation, boundary conditions) are described in Section 2. In Section 3, wind-tunnel experimental setups and model validation are conducted. The issues of $R e$-independence of indoor airflow and pollutant dispersion are discussed qualitatively and quantitatively in Section 4. Finally, Section 5 summarizes this study.

\section{Methodology}

\subsection{Physical model}

Figure 1 shows the physical model of this study, an isolated building with cross ventilation, where a pollution source with dimension of $0.2 \mathrm{~m} \times 0.2 \mathrm{~m} \times 0.1 \mathrm{~m}$ is located in the ground center. Two open windows $(W \times W)$ are on the windward and leeward sides of the cubic building $(L \times L \times$ $H)$, respectively. The fresh air always flows into the room through the windward window, and the foul air flows out through the leeward window.
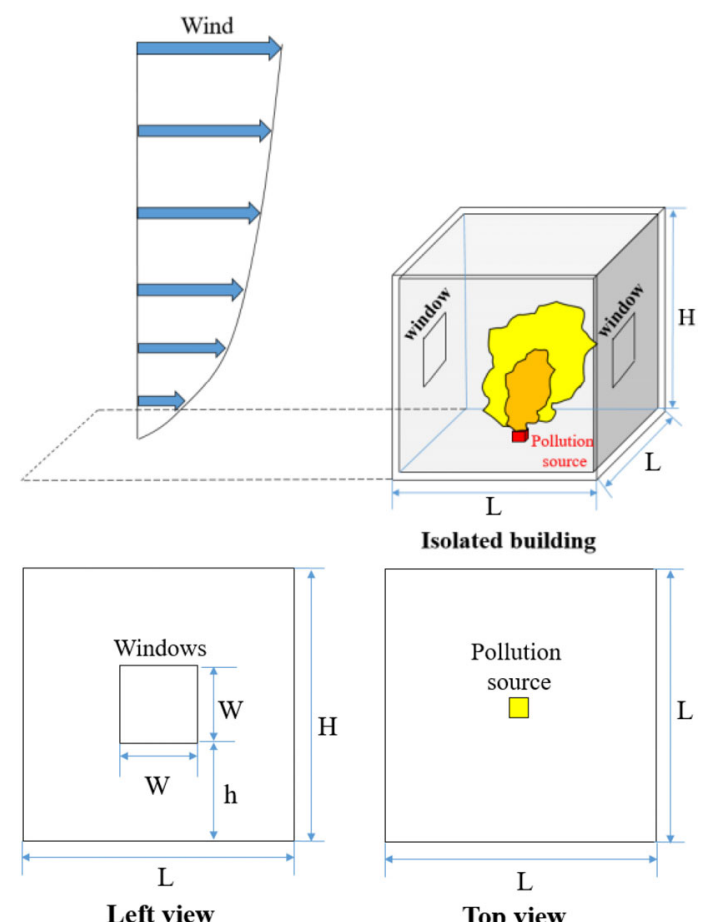

Isolated building

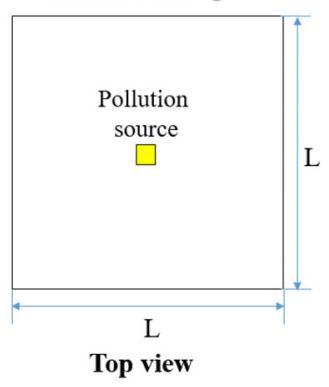

Fig. 1 Physical model
To ensure a good replication of outdoor and indoor flow structures and pollutant dispersion for the above physical model with reduced-scale model, the relevant $R e$ number should be greater than a certain critical value, i.e. $R e$-independence similarity must be followed. Generally speaking, building height Reynolds number $\left(R e_{\mathrm{H}}\right)$ is usually adopted to characterize the outdoor flow structures around the building (Uehara et al. 2003), which is defined as follows,

$R e_{\mathrm{H}}=U_{\mathrm{H}} H / v$

Similarly, the window Reynolds number $\left(R e_{\mathrm{w}}\right)$ is used as the characteristic $R e$ for indoor flow and dispersion (Dai et al. 2019), defined by,

$R e_{\mathrm{w}}=U_{\mathrm{w}} W / v$

where, $H$ is the building model height $(\mathrm{m}) ; W$ is the characteristic length of the window $(\mathrm{m}) ; U_{\mathrm{H}}$ represents the undisturbed wind speed in the upwind direction at the building height $(\mathrm{m} / \mathrm{s}) ; U_{\mathrm{w}}$ represents the mean velocity through the open window $(\mathrm{m} / \mathrm{s}) ; v$ is the kinematic viscosity of air $\left(=1.48 \times 10^{-5} \mathrm{~m}^{2} / \mathrm{s}\right)$. In this study, $U_{\mathrm{H}}$ is changing from $0.1 \mathrm{~m} / \mathrm{s}$ to $6 \mathrm{~m} / \mathrm{s}$, and the corresponding $R e_{\mathrm{H}}, U_{\mathrm{w}}$ and $R e_{\mathrm{w}}$ are shown in Table 2.

\subsection{Numerical model}

\subsubsection{Governing equations}

The dimensionless governing equations to describe the outdoor and indoor airflow and pollutant dispersion (without considering the thermal effect), are as follows, including the continuity equation in incompressible form (Uehara et al. 2003; Shu et al. 2020),

$\frac{\partial u_{i}^{\prime}}{\partial x_{i}^{\prime}}=0$

the RANS equation,

$\frac{\partial\left(u_{j}^{\prime} u_{i}^{\prime}\right)}{\partial x_{j}^{\prime}}=-\frac{\partial p^{\prime}}{\partial x_{i}^{\prime}}+\left(\frac{1}{R e}+\mu_{\mathrm{t}}^{\prime}\right) \frac{\partial^{2} u_{i}^{\prime}}{\partial x_{j}^{\prime 2}}+S_{\mathrm{m}}^{\prime}$

and the species transport equation for a passive scalar,

$\frac{\partial\left(u_{j}^{\prime} c_{i}^{\prime}\right)}{\partial x_{j}^{\prime}}=\frac{1}{R e}\left(\frac{1}{S c}+\frac{\mu_{\mathrm{t}}^{\prime}}{\mu^{\prime} S c_{\mathrm{t}}}\right) \frac{\partial^{2} c_{i}^{\prime}}{\partial x_{j}^{\prime 2}}+S_{c}^{\prime}$

Here, the superscripts (') indicates the dimensionless version of the relative variables. The dimensionless primitive variables are defined through the use of appropriate reference quantities, as follows, 
Table 2 Reynolds numbers ( $R e_{\mathrm{H}}$ and $\left.R e_{\mathrm{w}}\right)$ corresponding to characteristic velocities $\left(U_{\mathrm{H}}\right.$ and $\left.U_{\mathrm{w}}\right)$

\begin{tabular}{|c|c|c|c|c|c|c|c|}
\hline$U_{\mathrm{H}}(\mathrm{m} / \mathrm{s})$ & $R e_{\mathrm{H}}$ & $U_{\mathrm{w}}(\mathrm{m} / \mathrm{s})$ & $R e_{\mathrm{w}}$ & $U_{\mathrm{H}}(\mathrm{m} / \mathrm{s})$ & $R e_{\mathrm{H}}$ & $U_{\mathrm{w}}(\mathrm{m} / \mathrm{s})$ & $R e_{\mathrm{w}}$ \\
\hline 0.1 & 2,054 & 0.064 & 513 & 2.2 & 45,189 & 1.469 & 11751 \\
\hline 0.2 & 4,108 & 0.129 & 1030 & 2.4 & 49,297 & 1.607 & 12854 \\
\hline 0.4 & 8,216 & 0.266 & 2127 & 2.8 & 57,514 & 1.862 & 14894 \\
\hline 0.6 & 12,324 & 0.406 & 3230 & 3.2 & 65,730 & 2.123 & 16980 \\
\hline 0.8 & 16,432 & 0.541 & 4320 & 3.6 & 73,946 & 2.387 & 19092 \\
\hline 1.0 & 20,541 & 0.676 & 5410 & 4.0 & 82,162 & 2.651 & 21211 \\
\hline 1.2 & 24,649 & 0.810 & 6480 & 4.4 & 90,378 & 2.914 & 23311 \\
\hline 1.4 & 28,757 & 0.943 & 7540 & 4.8 & 98,595 & 3.176 & 25404 \\
\hline 1.6 & 32,865 & 1.075 & 8599 & 5.2 & 106,811 & 3.444 & 27554 \\
\hline 1.8 & 36,973 & 1.206 & 9640 & 5.6 & 115,027 & 3.714 & 29710 \\
\hline 2.0 & 41,081 & 1.334 & 10600 & 6.0 & 123,243 & 3.981 & 31854 \\
\hline
\end{tabular}

$x_{i}^{\prime}=\frac{x_{i}}{L}, u_{i}^{\prime}=\frac{u_{i}}{U_{0}}, p^{\prime}=\frac{p}{\rho U_{0}^{2}}, c_{i}^{\prime}=\frac{c_{i}}{c_{0}}, \mu^{\prime}=\frac{\mu}{\rho U_{0} L}$,

$\mu_{\mathrm{t}}^{\prime}=\frac{\mu_{t}}{\rho U_{0} L}, \rho^{\prime}=\frac{\rho}{\rho}=1, R e=\frac{\rho U_{0} L}{\mu}=\frac{1}{\mu^{\prime}} ; S c=\frac{v}{\alpha}$

The dimensionless momentum and species source terms are:

$S_{\mathrm{m}}^{\prime}=\frac{L}{U_{0}^{2}} S_{\mathrm{m}}, S_{\mathrm{c}}^{\prime}=\frac{L}{U_{0} c_{0}} S_{\mathrm{c}}$

$u$ is the time-averaged velocity; $U_{0}$ is the reference velocity $\left(=U_{\mathrm{H}}\right) ; L$ is the reference length (= $H$ for outdoor flow, $=W$ for indoor flow); $\rho$ is the air density; $p$ is the pressure; $c_{i}$ is the mass fraction of specie $i$; $c_{0}$ is the mass fraction of pollution source; $\alpha$ is the molecular diffusivity; $\mu$ and $\mu_{\mathrm{t}}$ are the kinetic viscosities; $S c_{\mathrm{t}}$ is the turbulent Schimdt number $(=0.7)$ (Tao 2001) and $S_{\mathrm{c}}$ denotes the pollution source term. Model validation (Section 3 ) has proved that the RNG $k-\varepsilon$ turbulence model can better predict the characteristics of the indoor airflow and pollutant dispersion, including the $k$ (turbulent kinetic energy) and $\varepsilon$ (the dissipation rate of turbulent kinetic energy) equations proposed by Yakhot and Orszag (1986). The nondimensionalization of $k$ and $\varepsilon$ equations can be referred to Shu et al. (2020).

\subsubsection{Computational domain and grid generation}

According to Tomanaga et al. (2008), the computational domain and its dimension are shown in Figure 2, where the inlet, top, right and left planes are all $6 \mathrm{H}$ away from the corresponding surfaces of building model, while the outlet plane is $18 \mathrm{H}$ away from the building leeward surface. The computational domain was discretized by Ansys ICEM 14.5 by structured grids with variable sizes: the finest grid resolution with the cell magnitude of $2-5 \mathrm{~mm}$ inside the indoor scale, a coarser resolution close to the building and ground surfaces with the magnitude of $0.5-1 \mathrm{~cm}$, and a gradually coarse mesh from the building and ground surfaces to the domain boundaries by using the increasing ratio of 1.121.16. After grid independence analysis, the total grid number is 5.8 million. Figure 3 shows the details of mesh generation inside and around the building on the vertical center plane, where the vertical measuring lines $\mathrm{A}-\mathrm{F}$ are marked.

\subsubsection{Boundary conditions and solution method}

The boundary conditions for the numerical models are summarized in Table 3.

(1) Inlet and outlet boundaries

The exponential velocity profile is imposed on the inlet

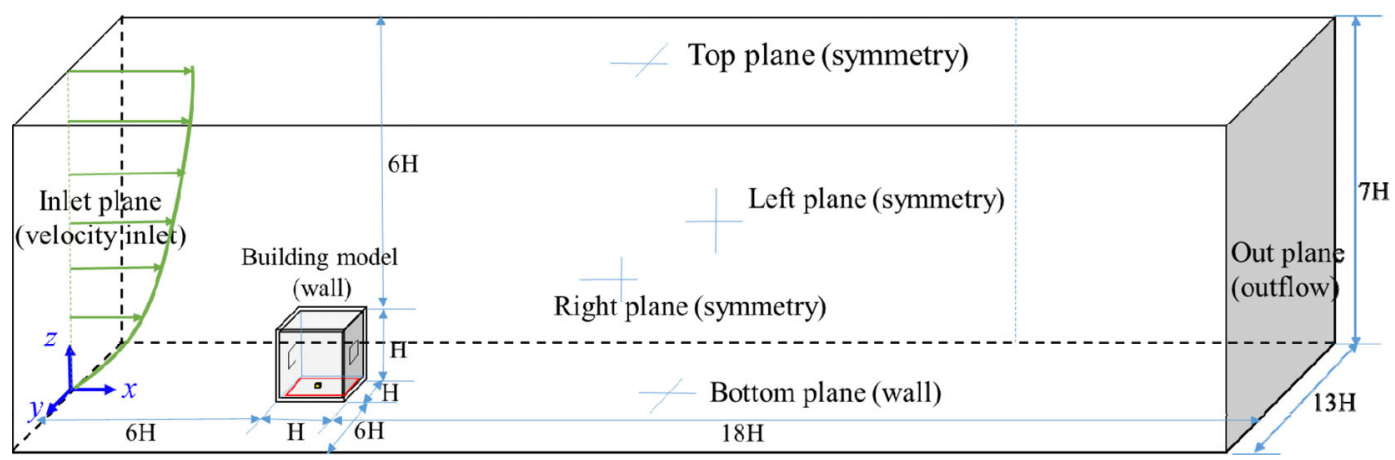

Fig. 2 Computational domain and boundary conditions 




Fig. 3 Mesh generation and measuring lines

plane of the computational domain, given as follows,

$\frac{U_{z}}{U_{\mathrm{H}}}=\left(\frac{z}{H}\right)^{\alpha}$

where, $U_{z}$ and $U_{\mathrm{H}}$ are the velocities at the height $z$ and reference height $H$, respectively; $\alpha$ is the wind profile exponent $(=0.19)$. The initial conditions for turbulent kinetic energy $k$ and its dissipation rate $\varepsilon$ are as follows,
$k=\frac{3}{2}(U I)^{2}$

$\varepsilon=c_{\mu}^{3 / 4} \frac{k^{3 / 2}}{l}$

where, $I$ is the turbulent intensity; $l$ is the turbulence length scale, equal to $0.07 L$ ( $L$ is the characteristic length of the inlet plane); $c_{\mu}(=0.09)$ is an empirical constant. The outflow boundary condition is imposed on the outlet plane where the first derivative for all flow variables along flow direction equals 0 .

(2) Top, left and right planes

Since the top, left and right planes are far away from the building, it can be assumed that they are not affected by the building wall effect, so the symmetry boundary conditions are more appropriate.

(3) Ground and building walls

The no-slip boundary condition and zero-gradient condition for velocity and species are assumed at ground and building walls, respectively. The standard wall functions (SWFs) proposed by Launder and Spalding (1974) are applied to deal with the flow and heat transfer near the solid surfaces for the RNG $k-\varepsilon$ model.

(4) Pollution source

In the reduced-scale model, the mixture of $\mathrm{SF}_{6}$ and nitrogen as the tracer gas is used to simulate the pollutants releasing from the indoor scale. The release rate $\left(Q_{e}\right)$ of the

Table 3 The boundary conditions for the numerical models

\begin{tabular}{|c|c|c|c|c|}
\hline Boundary conditions & Velocity & $k$ & $\varepsilon$ & Pollutant concentration \\
\hline Inlet plane & Velocity profile & $\frac{3}{2}(U(z) I(z))^{2}$ & $C_{\mu}^{3 / 4} \frac{k^{3 / 2}}{l}$ & 0 \\
\hline Outlet plane & $\begin{array}{c}\frac{\partial u}{\partial x}=0 \\
v=w=0\end{array}$ & $\frac{\partial k}{\partial x}=0$ & $\frac{\partial \varepsilon}{\partial x}=0$ & $\frac{\partial c}{\partial x}=0$ \\
\hline Central plane & $\begin{array}{c}\frac{\partial u}{\partial y}=0 \\
\frac{\partial w}{\partial y}=0 \\
v=0\end{array}$ & $\frac{\partial k}{\partial y}=0$ & $\frac{\partial \varepsilon}{\partial y}=0$ & $\frac{\partial c}{\partial y}=0$ \\
\hline Top plane & $\begin{array}{c}\frac{\partial u}{\partial z}=0 \\
\frac{\partial v}{\partial z}=0 \\
w=0\end{array}$ & $\frac{\partial k}{\partial z}=0$ & $\frac{\partial \varepsilon}{\partial z}=0$ & $\frac{\partial c}{\partial z}=0$ \\
\hline Right plane & $\begin{array}{c}\frac{\partial u}{\partial y}=0 \\
\frac{\partial w}{\partial y}=0 \\
v=0\end{array}$ & $\frac{\partial k}{\partial y}=0$ & $\frac{\partial \varepsilon}{\partial y}=0$ & $\frac{\partial c}{\partial y}=0$ \\
\hline Bottom plane & SWFs & 0 & 0 & 0 \\
\hline Ground/building walls & SWFs & 0 & 0 & 0 \\
\hline Pollutant source & 0 & 0 & 0 & Constant \\
\hline
\end{tabular}


pollution source (with the volume fraction of $\mathrm{SF}_{6}$ being 0.25 ) is $0.025 \mathrm{~g} /\left(\mathrm{m}^{3} \cdot \mathrm{s}\right)$.

The governing equations are discretized by the finite volume method (FVM), and solved by the commercial software Fluent 14.5. The SIMPLE scheme is adopted for handling the velocity-pressure coupling (Patankar 1980; Tao 2001), and the second order upwind scheme (Tao 2001) is applied for the spatial discretization of the convective term. The iteration convergence criterion is the residuals of the dependent variables being less than $10^{-5}$.

\section{Wind-tunnel experimental setups and model validation}

The wind-tunnel tests were carried out in Environmental
Wind Tunnel Laboratory at University of Shanghai for Science and Technology (EWT Lab at USST), which is an open-circle atmospheric boundary layer wind tunnel with the test section dimensions of $2.5 \mathrm{~m}$ (width) $\times 1.8 \mathrm{~m}$ (height) $\times$ $25 \mathrm{~m}$ (length). The wind speeds can be adjusted from $0.1 \mathrm{~m} / \mathrm{s}$ to $20 \mathrm{~m} / \mathrm{s}$ continuously, and six Irwin-type spires and roughness elements with different sizes are regularly arranged in the development section ( $15 \mathrm{~m}$ long) to generate the scaled profiles of atmospheric boundary layer (ABL) flow (Figure 4). The building model with reduced-scale ratio of 1:10 is placed at the center of a circular turntable with a diameter of $2 \mathrm{~m}$ in the testing section.

The TSI IFA300 3D constant temperature hot-film anemometer with a high accuracy of $\pm 0.1 \%$ was used to measure the mean velocity and turbulence parameters. After

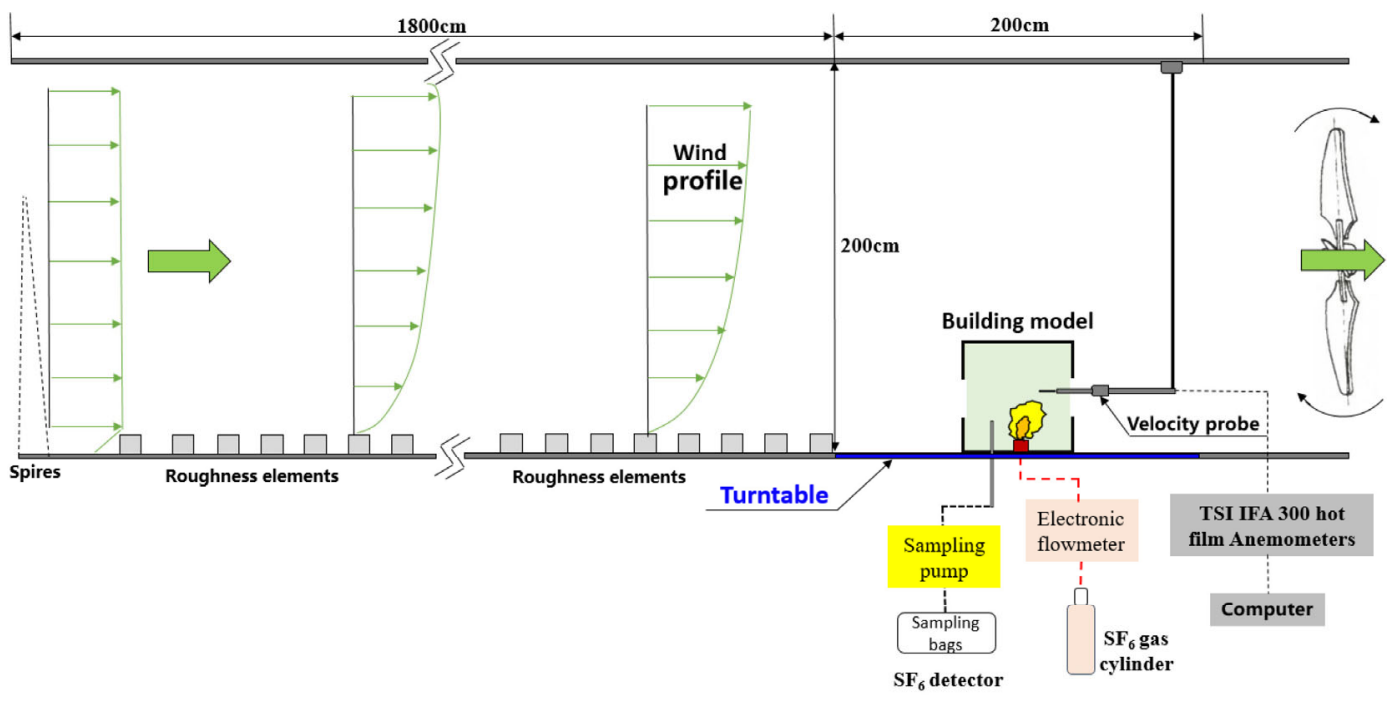

(a) Wind tunnel experimental setups

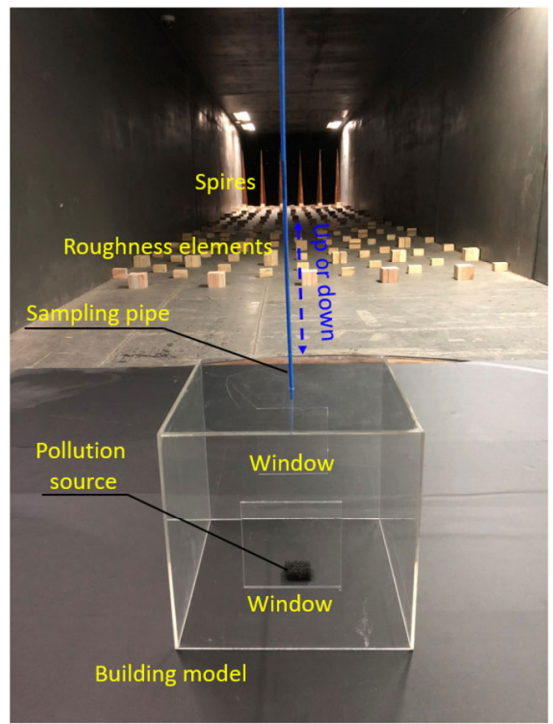

(b) Building model

Fig. 4 Wind-tunnel experimental models and setups 
the tracer gases releasing, the sampling tubes $(D=2 \mathrm{~mm})$ connected with the sampling pumps (sampling rate of $150 \mathrm{~mL} / \mathrm{min}$ ) collect them into sampling bags at different locations (Figures 4 (a) and (b)). DB-600T SF 6 quantitative detector with a measuring accuracy of $\pm 0.1 \mathrm{ppm}$ was used to measure the tracer gas concentrations. The normalized concentration $K$ is defined as follows,

$K=\frac{C_{\mathrm{r}} U_{\mathrm{H}} H^{2}}{C_{\mathrm{e}} Q_{\mathrm{e}}}$

where $C_{\mathrm{r}}$ is the volume fraction of the measured $\mathrm{SF}_{6}(\mathrm{ppm}) ; C_{\mathrm{e}}$ is the volume fraction of the pollutant source $\left(=2.5 \times 10^{5} \mathrm{ppm}\right)$; $U_{\mathrm{H}}=6 \mathrm{~m} / \mathrm{s}$.

The wind-tunnel experimental data (WT data) was used to validate the established numerical model, where three high-Re turbulence models were adopted, namely standard, RNG, and realizable $k-\varepsilon$ (SKE, RNG, RLKE) turbulence models. In Figure 5(a), the normalized velocity $\left(U / U_{0}\right)$ distributions along indoor vertical center line $E$ measured by wind-tunnel tests are first compared with the numerical simulation results. It can be noted that RNG model shows a better agreement with the WT data (mean deviation less than about 6\%) than the other two turbulence models, although the results of the three turbulence models show the same trend, and the difference are not significant. Then the comparison of the normalized concentration $(K)$ distributions along vertical line E measured by wind-tunnel measurements with that of the numerical models (SKE, RNG, RLKE) is shown in Figure 5(b). We find that the pollutant concentration $K$ decreases sharply with the increase of height due to the increasing distance from the pollution source and the effect of cross ventilation in the middle. The numerical results from RNG model get much closer to the WT data distributions; however, the results from SKE and RLKE models with $S c_{t}=0.7$ underestimate the pollutant concentrations. In Figures 5(c) and (d), we compared the measured normalized velocity $\left(U / U_{0}\right)$ and pollutant concentrations $(K)$ along outdoor vertical measuring line $\mathrm{C}$ with three numerical simulation results, respectively. The similar conclusion of RNG model performing better can be drawn. Although due to the limits of the RANS models, it is more difficult to accurately predict the reverse flow inside and behind the building compared with large eddy simulation (LES), many previous studies have shown that RNG model can still provide a compromise between the prediction accuracy and calculation resource cost (Ramponi and Blocken 2012; Cui et al. 2014; Ai and Mak 2014b; Perén et al. 2015; Cui et al. 2017; van Hooff et al. 2017). Therefore, the RNG model can perform better for simulating the indoor and outdoor airflow and pollutant dispersion, which will be applied to numerically study the following $R e$-independence issues.


(a) $U / U_{0}$ along indoor vertical center line $\mathrm{E}$

(b) $K$ along indoor vertical center line $\mathrm{E}$
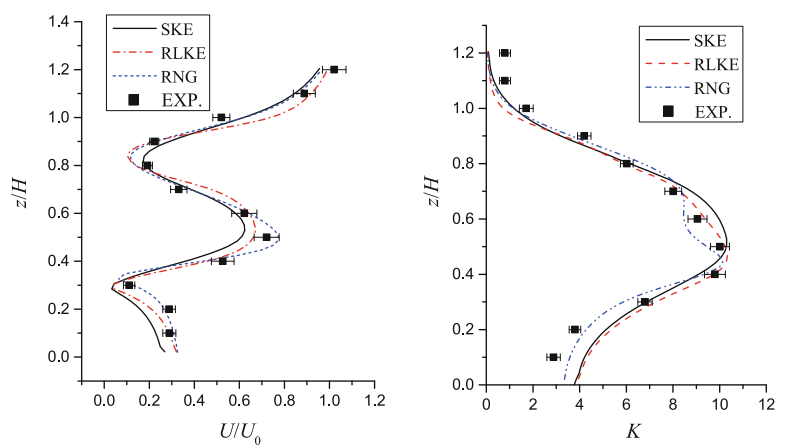

$\begin{array}{lll}\text { (c) } U / U_{0} \text { along outdoor vertical measuring line } \mathrm{C} & \text { (d) } K \text { along outdoor vertical measuring line } \mathrm{C}\end{array}$

Fig. 5 Model validation (standard, realizable and RNG $k$ - $\varepsilon$ models vs. WT data)

\section{Results and discussion}

4.1 Flow characteristics in and around the isolated building with varying $R e$

Due to the different characteristic velocities and geometry scales, $R e_{\mathrm{H}}$ (Eq.(1)) and $R e_{\mathrm{w}}$ (Eq.(2)) are adopted to characterize the outdoor and indoor flow structures, respectively. Figures 6(a)-(d) show the distributions of dimensionless velocity $U / U_{0}$ along vertical lines A-D (outdoor) with varying $R e_{\mathrm{H}}$. By comparing the velocity distributions along different vertical lines, several features can be observed. For the outdoor flow structures, the $U / U_{0}$ variations with $R e_{\mathrm{H}}$ at four typical locations are monitored: the windward side (line A), rooftop (line $B$ ) and leeward side (lines $C$ and $D$ ) of the building. Firstly, from Figure 6(a), we find that when $R e_{\mathrm{H}}$ is small and $z / H<0.3$ along line A (in the corner vortex), the variation of dimensionless velocity changes a lot with the increasing $R e_{\mathrm{H}}$, however, this variation becomes mild when $R e_{\mathrm{H}}$ is over a certain value. And when $z / H>0.3$ (away from the corner vortex), the velocity distributions almost coincide, indicating that the flow is independent of $R e_{\mathrm{H}}$. Secondly, as shown in Figure 6(b), the dimensionless velocity distributions along line $\mathrm{B}$ are nearly unchanged with the increasing $R e_{\mathrm{H}}$. Since the lower part of line $\mathrm{B}$ is not located in the separation vortex in front of the rooftop (see in Figure 10), all points along line $\mathrm{B}$ are easy to enter into the flow Re- 
independence regime. Thirdly, Figure 6(c) shows that the flow structures along line $\mathrm{C}$ can be divided into four parts according to its portions relative to the window: below the window $(z / H<0.33)$, in the window level $(0.33<z / H<$ $0.66)$, above the window $(0.66<z / H<1)$ and above the rooftop $(z / H>1)$. It can be seen that the flow structures below the window is most affected by $R e_{\mathrm{H}}$ (influenced by the building wake and near wall effects), followed by the part in the window level (large velocity gradient), and then the part above the window (building wake effect). However, when $z / H>1$ (above the rooftop), the flow can enter into the $R e$-independence easily because it is far away from the wall and vortex, the viscous force can be negligible. Moreover, the similar characteristics of the velocity distribution with varying $R e_{\mathrm{H}}$ along line $\mathrm{D}$ (also behind the building as line $\mathrm{C}$ ) can be found in Figure 6(d). But the difference is that the dimensionless velocity distributions most affected by $R e_{\mathrm{H}}$ along line $\mathrm{D}$ is in the window level (the relatively large velocity gradient and flow separation effect), followed by the part above the window (flow separation effect), and then the part below the window (away from the building wake).

The distributions of dimensionless velocity $U / U_{0}$ along indoor vertical lines $\mathrm{E}$ (center vertical line) and $\mathrm{F}$ (close to the leeward window) with varying $R e_{\mathrm{w}}$ are described in Figures 6(e) and (f). It is noted that the characteristics of dimensionless velocity distribution and variation trend along these two vertical lines are basically the same, where the vertical line can also be divided into three parts based on the flow structure characteristics: below the window $(z / H<$ $0.33)$, in the window level $(0.33<z / H<0.66)$ and above the

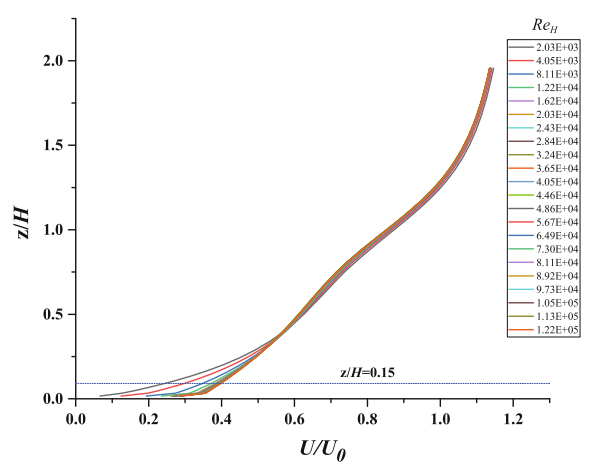

(a) $U / U_{0}$ along line $\mathrm{A}$

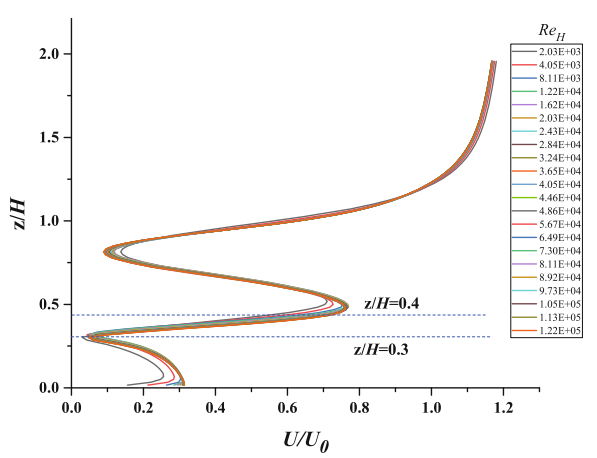

(c) $U / U_{0}$ along line $\mathrm{C}$

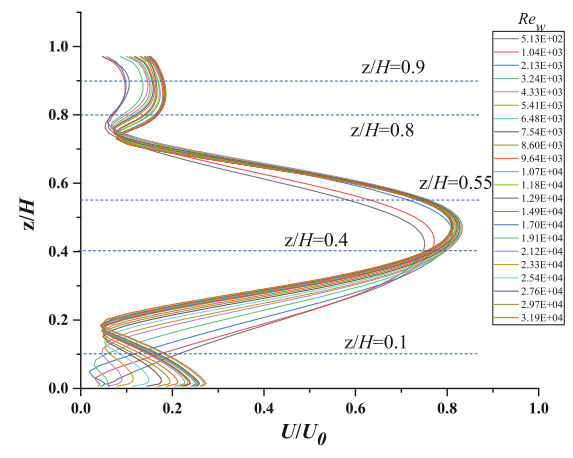

(e) $U / U_{0}$ along line $\mathrm{E}$

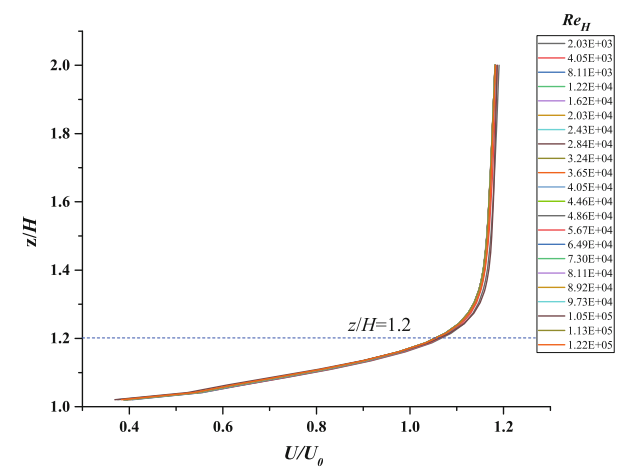

(b) $U / U_{0}$ along line $\mathrm{B}$

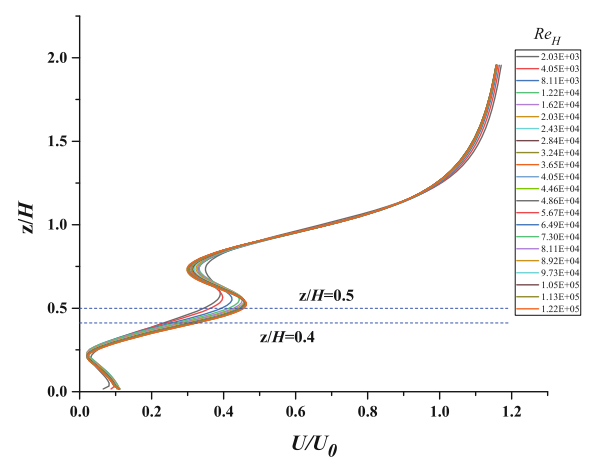

(d) $U / U_{0}$ along line $\mathrm{D}$

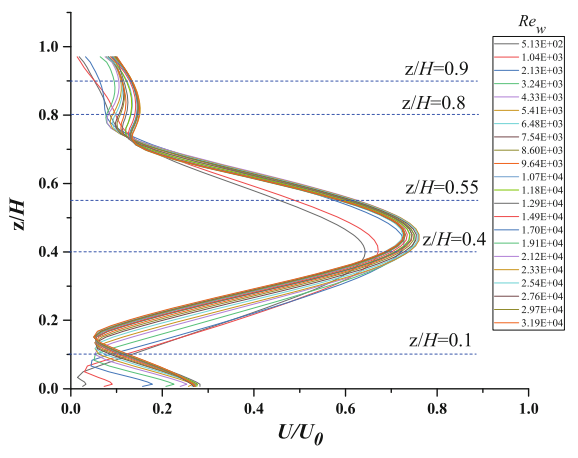

(f) $U / U_{0}$ along line $\mathrm{F}$

Fig. 6 Distributions of dimensionless velocity $U / U_{0}$ along vertical lines (outdoor and indoor) with varying $\left(R e_{\mathrm{H}}\right) R e_{\mathrm{w}}$ 
window $(z / H>0.66)$. Firstly, we note that because of the stronger wall effect close to the ground or building wall, the flow structures below and above the window are more dependent on $R e_{w}$, followed by the part in the window level. That is to say the flow structures at the window level can enter the $R e$-independence regime more easily. Secondly, as the whole, when $R e_{\mathrm{w}}$ is relatively small, the dimensionless velocity distributions change obviously with $R e_{\mathrm{w}}$ increasing. Until $R e_{\mathrm{w}}$ is over a certain value (critical value), this variation along the whole line gradually becomes mild, in other words, indicating that the indoor flow structure can be considered to enter the $R e$-independence regime. Thirdly, compared to the velocity distributions around the building (Figures 6 (a)-(d)), it is more clearly difficult for the indoor flow structures to enter the $R e$-independence regime, resulting from the flow in a narrow space being subject to a larger wall viscous forces.

From the above qualitative analysis, it can be concluded that: first, it needs different Reynolds numbers $\left(R e_{\mathrm{w}}\right.$ and $\left.R e_{\mathrm{H}}\right)$ to characterize the flow structures in and around the isolated building. Second, due to the different relative strength of viscous and inertial forces, it needs different critical values of $R e$ to ensure the flow at different locations to enter the $R e$-independence regime. Third, it is easier for the flow around the building to enter the $R e$-independence regime than the indoor flow structures, resulting from being subject to a relative small viscous force in a semi-infinite space. Furthermore, there should exist the critical $R e_{\mathrm{H}}$ and $R e_{\mathrm{w}}$, over which the outdoor and indoor flows at all locations will enter the Re-independence regime. The following section will determine the thresholds.

\subsection{Determining critical Reynolds numbers $\left(R e_{H, \text { crit }}\right.$ and $\left.R e_{\mathrm{w}, \text { rit }}\right)$}

To quantitatively determine the critical values of $\operatorname{Re}\left(R e_{\mathrm{H}, \text { crit }}\right.$ and $\left.R e_{w, c r i t}\right)$, we firstly propose the following quantity, namely the ratio of relative changes $(R R C)$, for judging whether the outdoor flow structures can be regarded in the $R e$-independence regime (Cui et al. 2014):

$R R C=\frac{\Delta V / V}{\Delta R e_{\mathrm{H}} / R e_{\mathrm{H}}}$

where $V$ is the dimensionless characteristic velocity, $\Delta V$ is its change corresponding to the change in $R e_{\mathrm{H}}, \Delta R e_{\mathrm{H}}$. However, the quantity of $R R C$ is very sensitive to the dramatic changes in velocity (velocity gradient or direction), such as the locations near the indoor jet flow through the window, the separation flow behind the building. Given this, another quantity of $D R$ (difference ratio) of the dimensionless velocity was specified as follows,
$D R=\left|\frac{U_{R e_{i}}-U_{R e_{j}}}{U_{R e_{i}}}\right|$

where $U_{R e_{i}}$ and $U_{R e_{i}}$ denote the mean dimensionless velocities corresponding to two adjacent $R e$ numbers, $R e_{i}$ and $R e_{j}$, respectively. Unlike the $R R C, D R$ is not particularly sensitive to the varying velocity, and it would be also reasonable to use $D R-R e$ to describe the $R e$ independence of flow structures in a physical sense. Generally, the relative variation less than $5 \%$ can often be neglected in conventional engineering practice. Thus, $R R C$ or $D R \leq 5 \%$ can be regarded as the criterion of $R e$-independence of the flow structures in this study.

\subsubsection{Critical $\operatorname{Re}_{H}\left(\operatorname{Re}_{H, \text { crit }}\right)$ for outdoor flow}

Figures $7(\mathrm{a})$ and (b) show the mean $R R C$ vs. $R e_{\mathrm{H}}$ along line $\mathrm{A}(z / H$ from 0 to 0.15$)$ and along line $\mathrm{B}(z / H$ from 1 to 1.2$)$, respectively. Firstly, from Figure $7(\mathrm{a})$, we note that when $R e_{\mathrm{H}}<14,000, R R C$ will decrease sharply from $25 \%$ down to $5 \%$ with the increasing $R e_{\mathrm{H}}$, indicating this region is strongly $R e$ dependent, i.e., the flow structures change significantly with $R e$. However, when $R e_{\mathrm{H}}>14,000$, the $R R C$ almost never changes with the increasing $R e_{\mathrm{H}}$, that is, the flow enters the $R e$-independence regime. With $R R C \leq 5 \%$ as the standard, we suggest that the mean critical value of $R e_{\mathrm{H}}, R e_{\mathrm{H}, \text { crit }}=$ 14,000 for $z / H$ from 0 to 0.15 along line A. Secondly, Figure7(b) show the mean $R R C$ at $z / H$ from 1 to 1.2 along line $\mathrm{B}$ was below the standard of $5 \%$, indicating that flows in this region could easily enter the Re-independence regime. That is because line B in this study is at the top center and out of the separation vertex in front of the build rooftop, which is different from the position of line $\mathrm{A}$ in reference (Cui et al. 2014). Thirdly, the distributions of mean $D R$ of dimensionless velocity with varying $R e_{\mathrm{H}}$ at $z / H$ from 0.3 to 0.4 along line $\mathrm{C}$ and $z / H$ from 0.4 to 0.5 along line $\mathrm{D}$ are shown in Figures 7(c) and (d), respectively. We note that the variation trend of the two figures is similar, that is, when the $R e_{\mathrm{H}}$ is small, there exists a strong $R e$-dependence flow ( $D R$ decreasing sharply with the increasing $R e_{\mathrm{H}}$ ), while $R e_{\mathrm{H}}$ exceeds a certain value, $D R$ no longer changes with the increase of $R e_{\mathrm{H}}$ (the flows enter the weak $R e$-dependence region). However, with the standard of $D R \leq 5 \%$, the suggested values of $R e_{\mathrm{H}, \text { rrit }}$ are 27,000 and 10,000 for the flows along lines $\mathrm{C}$ and $\mathrm{D}$, respectively, which is consistent with the results of the above qualitative analysis in Figures 6(c) and (d).

Combined with the above analysis in Figure 6, it can be concluded that due to the different relative strengths of the viscous force and inertia force, it requires different critical $R e$ numbers at different positions to ensure the flows enter the $R e$-independence regime. The critical $R e_{\mathrm{H}}$ is smaller at 


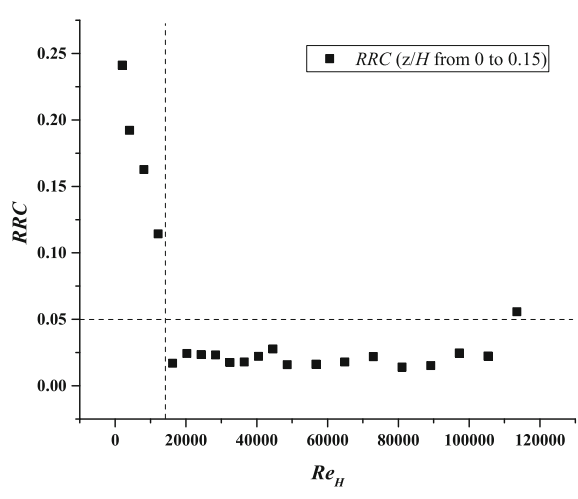

(a) $R R C$ vs. $R e_{\mathrm{H}}$ along line $\mathrm{A}$

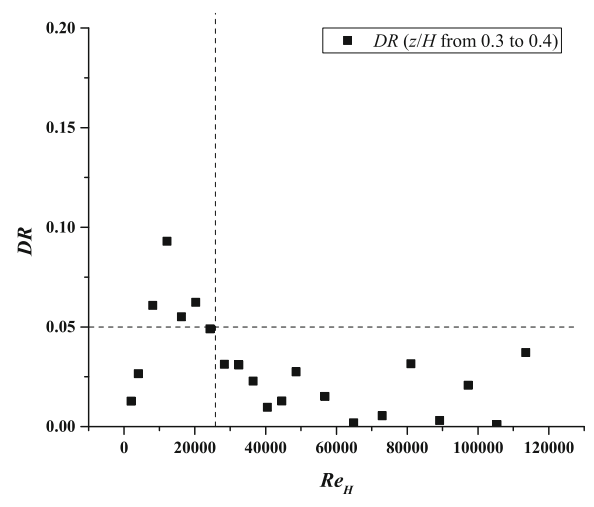

(c) $D R$ vs. $R e_{\mathrm{H}}$ along line $\mathrm{C}$

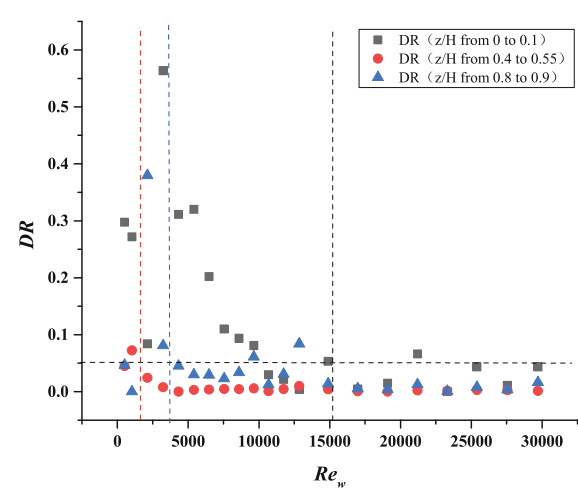

(e) $D R$ vs. $R e_{w}$ along line $\mathrm{E}$

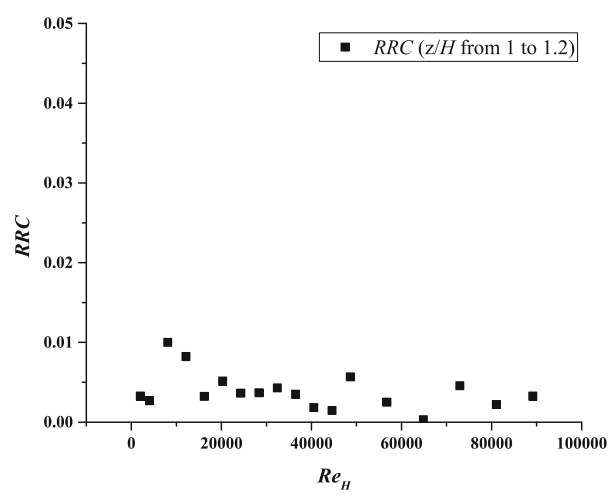

(b) $R R C v s . R e_{\mathrm{H}}$ along line $\mathrm{B}$



(d) $D R$ vs. $R e_{\mathrm{H}}$ along line $\mathrm{D}$

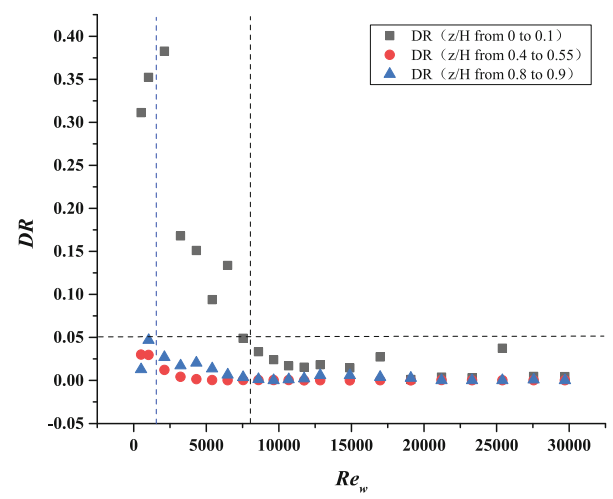

(f) $D R$ vs. $R e_{\mathrm{w}}$ along line $\mathrm{F}$

Fig. $7 D R$ or $R R C$ vs. $R e_{\mathrm{w}}\left(R e_{\mathrm{H}}\right)$ along different vertical measuring lines (Lines A-F) for medium window building model

the position where the viscous force can be ignored; otherwise, the larger critical $R e_{\mathrm{H}}$ is required. Therefore, the critical $R e_{\mathrm{H}}$ at the most unfavorable position should be selected as the optimal value to ensure the $R e$-independence of the flows within the whole areas of interest. In this study, the wake region on the leeward side of the building is the most unfavorable, thus $R e_{\mathrm{H}, \text { crit }}=27,000$ is recommended for the outdoor flow.

\subsubsection{Critical $R e_{w}\left(R e_{w, \text { crit }}\right)$ for indoor flow}

The distributions of mean $D R$ vs. $R e_{\mathrm{w}}$ at different positions along line $\mathrm{E}$ and line $\mathrm{F}$ are shown in Figures 7(e) and (f) (the medium window case), respectively, where three positions are selected: below the window $(z / H$ from 0 to 0.1$)$, at the window level $(z / H$ from 0.4 to 0.55$)$ and above the window ( $z / H$ from 0.8 to 0.9 ). From these two figures, we note that the flows at the window level are more easily to enter the $R e$-independence regime, followed by the position above the window, then that below the window. Firstly, Figure 7(e) shows almost all the $D R$ values at the window level are below the indicator of $D R<5 \%$, indicating that at the window level with relatively high jet flow and negligible viscous force, the flows require smaller $R e_{\mathrm{w}, \text { crit }}$ (about 2,000) to enter the $R e$-independence regime. Secondly, when $R e_{\mathrm{w}}>3,000$, 
most of the $D R$ values above the window are below the indicator of $D R<5 \%$ and change little with the increasing $R e_{\mathrm{w}}$, therefore, the $R e_{\mathrm{w}, \text { crit }}=3,000$ is suggested at this position. Moreover, we can find that the flows below the window are more difficult to enter the $R e$-independence regime, and require a lager $R e_{\mathrm{w}, \text { crit }}(=15,000)$ to ensure that $D R$ values will not change with the increasing $R e_{\mathrm{w}}$. In addition, there exist obviously strong $\left(R e_{\mathrm{w}}<15,000\right)$ and weak $\left(R e_{\mathrm{w}}>15,000\right)$ $R e$-dependence regions below the window, and the other two positions have no obvious strong $R e$-dependence regions. Figure 7(f) shows the similar distributions of $D R$ vs. $R e_{\mathrm{w}}$ at three positions along line $\mathrm{F}$ with that along line $\mathrm{E}$ in Figure $7(\mathrm{e})$. However, the flows along line $\mathrm{F}$ are easier to go to the $R e$-independence regime, and $R e_{\mathrm{w}, \text { crit }}(=8,000$, also below the window) is suggested. Through the above quantitative analysis, $R e_{\mathrm{w}, \text { crit }}=15,000$ is suggested as the critical value for the indoor air flows.

To further confirm whether $R e_{\mathrm{w}, \text { crit }}(=15,000)$ is related to window sizes, the other two numerical cases with different window sizes are examined, namely large $(4 \mathrm{~W} / 3)$ and small $(2 \mathrm{~W} / 3)$ window cases. Then the distributions of $D R$ vs. $R e_{\mathrm{w}}$ at different positions along lines $\mathrm{E}$ and $\mathrm{F}$ for large and small window cases are presented in Figures 8 and 9, respectively. Here, Figures 8(a) and (b) denote the similar variation trends of the $D R$ vs. $R e_{\mathrm{w}}$ along lines $\mathrm{E}$ and $\mathrm{F}$ with that in Figures $7(\mathrm{e})$ and (f). It shows that the air flows at the window level are easier to enter the $R e$-independence regime (all the $D R$ values below the indicator of $5 \%$ ), followed by the position above the window $\left(R e_{\mathrm{w}, \text { crit }}=2,500\right.$ suggested $)$, then that below the window where a larger $R e_{\mathrm{w}, \text { crit }}(=13,000)$ divides the flows into obvious strong and weak $R e$-dependence regions. In addition to the similar variation trend ( $D R$ vs. $R e_{\mathrm{w}}$ ) compared to Figures7 (e) and (f), Figures 9(a) and (b) have the following characteristics. Firstly, for the small window case, there are more $D R$ values above the indicator of $5 \%$ along line $\mathrm{E}$, where $R e_{\mathrm{w}, \text { crit }}=2,100$ is suggested at the window level, followed by 4,800 above the window, then about $R e_{\mathrm{w}, \text { crit }}=$ 14,000 is suggested below the window (the unfavorable position). Secondly, we note that the mean $R e_{\mathrm{w}, \text { crit }}$ at the window level increases slightly with the decrease of the window dimension, all the $D R$ values below the indicator of $5 \%$ in the medium and large window case, while in the small window case, $R e_{\mathrm{w}}>3,000$ can only make the flow enter into the $R e$-independence regime. Thirdly, to monitor the $D R$

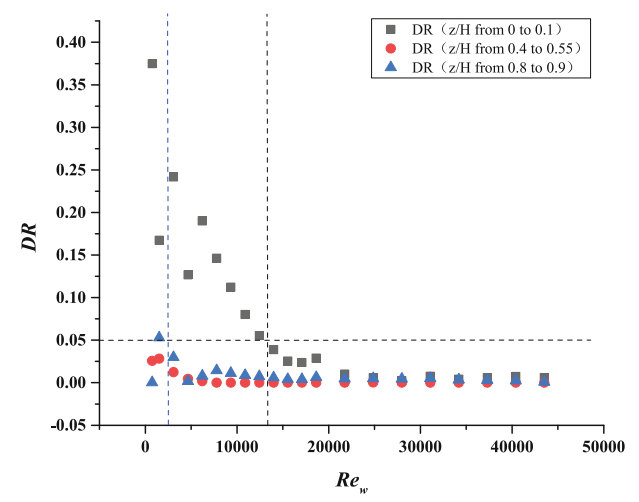

(a) $D R$ vs. $R e_{\mathrm{w}}$ along line $\mathrm{E}$

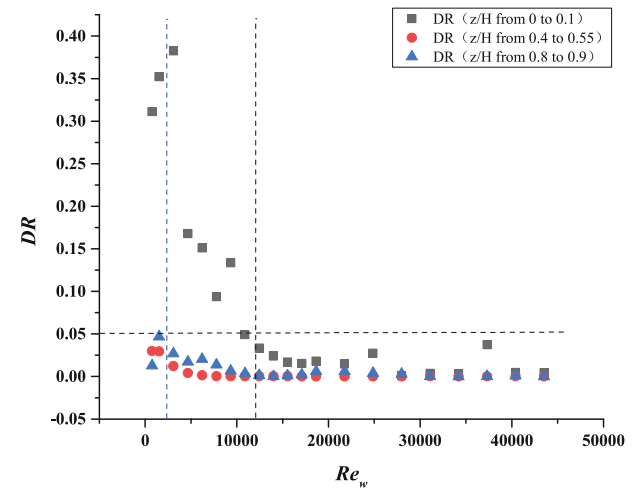

(b) $D R$ vs. $R e_{\mathrm{w}}$ along line $\mathrm{F}$

Fig. $8 D R$ vs. $R e_{\mathrm{w}}$ along different vertical measuring lines (Lines E-F) for large window building model

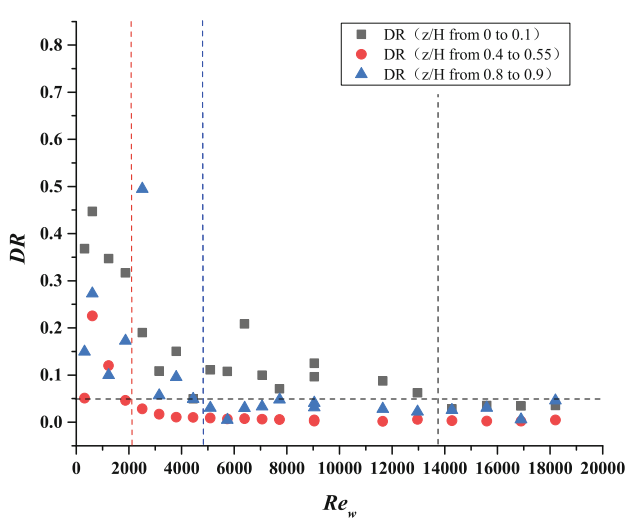

(a) $D R$ vs. $R e_{\mathrm{w}}$ along line $\mathrm{E}$

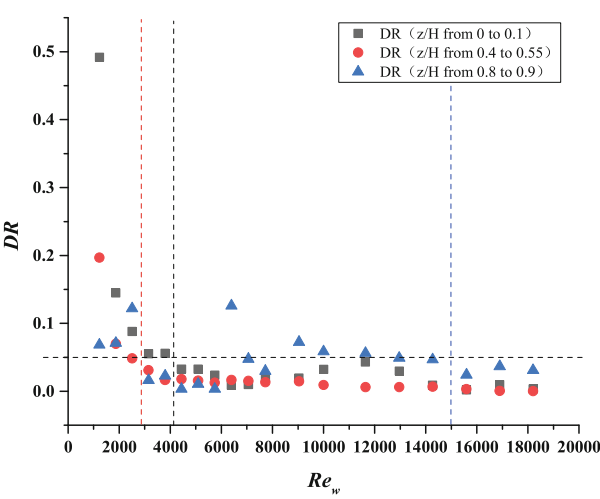

(b) $D R$ vs. $R e_{\mathrm{w}}$ along line $\mathrm{F}$

Fig. $9 D R$ vs. $R e_{\mathrm{w}}$ along different vertical measuring lines (Lines E-F) for small window building model 
value at the position of line $\mathrm{F}$, the flows above the window $\left(R e_{\mathrm{w}, \text { crit }}=15,000\right.$ suggested $)$ are more difficult to enter the $R e$-independence regime than the flow below the window $\left(R e_{w, \text { crit }}=4,100\right.$ suggested $)$ in the case of small window, which is obviously different with the former two cases.

Table 4 lists the corresponding critical $R e_{\mathrm{w}}\left(R e_{\mathrm{w}, \text { crit }}\right)$ values for the flow entering $R e$-independence regime at different positions along the monitoring lines $\mathrm{E}$ and $\mathrm{F}$ under three window size cases. Combined with the above analysis, the following conclusions can be further summarized. First, the $R e_{\mathrm{w}, \text { rrit }}$ values are position dependence in the room due to the different relative strengths of inertial force and wall viscous force. Second, under these three window size cases, the airflow near the window level is the easiest to enter the $R e$-independence regime, and the lager the window dimension is, the lower the Re-dependence is required. Third, The maximum $R e_{\mathrm{w}, \text { crit }}$ value appears in the vortex region near the wall at the upper or lower part of the window. Although the position is slightly different, the maximum values are all among $14,000-15,000$, therefore, the $R e_{w, \text { crit }}$ of 15,000 is suggested for indoor airflow and cross ventilation regardless of the window size.

\subsection{Further confirmation of critical $\operatorname{Re}_{\mathrm{w}}\left(\operatorname{Re}_{\mathrm{w}, \text { crit }}\right)$}

To further confirm the critical value of $R e_{\mathrm{w}}\left(R e_{\mathrm{w}, \text { crit }}=15,000\right)$, Figure 10 shows the contours of dimensionless velocity $\left(U / U_{0}\right)$ against the increasing $R e_{\mathrm{w}}$ numbers. The following conclusions can be drawn. First, when the $R e_{\mathrm{w}}<14,894$ (Figures 10 (a)-(e)), with the increase of $R e_{\mathrm{w}}$, the velocity distributions change significantly, especially in the lower part of the window, followed by the upper part of the window. The position at the window level is easier to enter the Reindependence regime, which is because the wind speed here is relatively high and the viscous force can be ignored compared with the inertial force. Second, when the $R e_{\mathrm{w}}>$ 14,894 , it can be found that with the $R e_{\mathrm{w}}$ increase, the velocity distributions at each part (window level, above and below the window level) will not change (Figures 10 (f)-(h)) yet, indicating that the indoor airflow enters the Re-independence regime when $R e_{\mathrm{w}}$ is over 14,894 . This is basically consistent with the varying trend of $D R$ value (Figures 7 (e) and (f)) along the indoor monitoring lines, which further confirms that the suggested $R e_{w, \text { crit }}$ for indoor flow structures entering $R e$-independence can be taken as 15,000.

\subsection{Re-independence for indoor pollutant dispersion}

To further confirm whether there is Re-independence regime for the diffusion of the indoor air pollutant within the reduced-scale building model. It means whether there is also a critical value of $R e_{\mathrm{w}}$, when $R e_{\mathrm{w}}$ is greater than this value, the dimensionless concentration distributions in the room will no longer change. Figure 11(a) gives the distributions of dimensionless concentration $K$ along indoor vertical center lines with varying $R e_{\mathrm{w}}$. It can be seen from the figure that the distribution of dimensionless concentration $K$ along with $R e_{\mathrm{w}}$ is similar to that of dimensionless velocity. On the whole, when $R e_{\mathrm{w}}$ is small, the dimensionless concentration $K$ changes significantly with the increase of $R e_{\mathrm{w}}$, especially the regions below the window $(0<z / H<0.33)$; with the increase of $R e_{\mathrm{w}}\left(R e_{\mathrm{w}}>4330\right)$, the distribution of $K$ above the window $(0.66<z / H<1)$ first enters the $R e$-independence regime. As $R e_{\mathrm{w}}$ continues to increase $\left(R e_{\mathrm{w}}>9640\right)$, the distribution of $K$ in the window level $(0.33<z / H<0.66)$ goes into $R e$-independence regime, and followed by the part below the window $(0<z / H<0.33)$. It can be seen from the above points that the part below the window $(0<z / H<$ $0.33)$ is the most unfavorable region for the dimensionless concentration $K$ to enter the Re-independence regime. As long as the unfavorable region can enter the $R e$-independence regime of pollutant diffusion, the other indoor locations can meet this requirement.

To determine the critical value that can ensure the Reindependence of indoor pollutant diffusion, Figure 11(b) shows the distributions of $K_{-} D R$ vs. $R e_{\mathrm{w}}$ at the most unfavorable position $(0<z / H<0.1)$. $K \_D R$ is defined to solve the variation of mean dimensionless concentration $K$ with $R e_{\mathrm{w}}$, as follows:

$$
K_{-} D R=\left|\frac{K_{R e_{i}}-K_{R e_{j}}}{K_{R e_{i}}}\right|
$$

where $K_{R e_{i}}$ and $K_{R e_{j}}$ denote the mean dimensionless

Table $4 R e_{\mathrm{w}, \text { crit }}$ values along Lines $\mathrm{E}$ and $\mathrm{F}$ under three window size cases

\begin{tabular}{|c|c|c|c|c|}
\hline \multicolumn{2}{|c|}{$R e_{\mathrm{w}, \text { crit }}$} & \multirow{2}{*}{$\frac{z / H \text { from } 0 \text { to } 0.1}{13,800}$} & \multirow{2}{*}{$\frac{z / H \text { from } 0.4 \text { to } 0.55}{2,100}$} & \multirow{2}{*}{$\frac{z / H \text { from } 0.8 \text { to } 0.9}{4,800}$} \\
\hline Small window & Line $\mathrm{E}$ & & & \\
\hline Sind & Line F & 4,200 & 2,800 & 15,000 \\
\hline \multirow{2}{*}{ Moderate window } & Line $\mathrm{E}$ & 15,000 & 1,800 & 3,800 \\
\hline & Line F & 8,000 & - & 1,800 \\
\hline \multirow{2}{*}{ Large window } & Line $\mathrm{E}$ & 13,500 & - & 2,500 \\
\hline & Line $F$ & 12,000 & - & 2,500 \\
\hline
\end{tabular}






(a) $R e_{\mathrm{w}}=513$

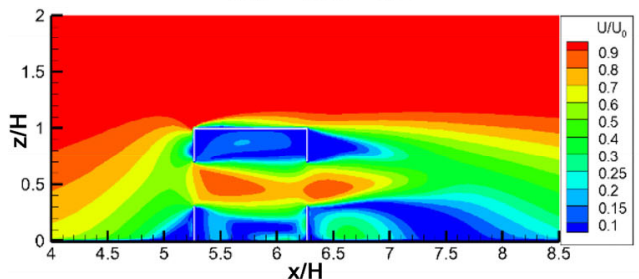

(c) $R e_{\mathrm{w}}=5410$



(e) $R e_{\mathrm{w}}=11751$



(g) $R e_{\mathrm{w}}=16980$

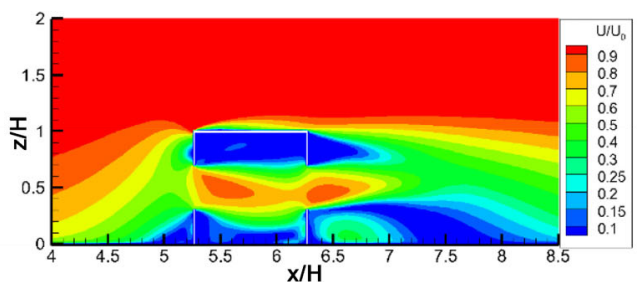

(b) $R e_{\mathrm{w}}=2127$

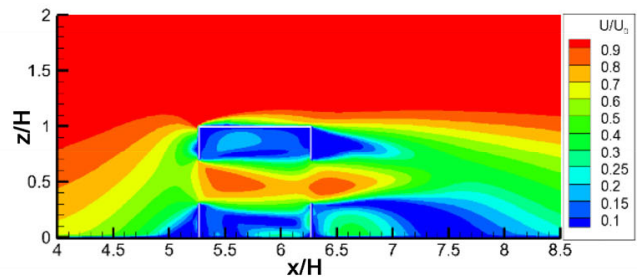

(d) $R e_{\mathrm{w}}=8599$



(f) $R e_{\mathrm{w}}=14894$

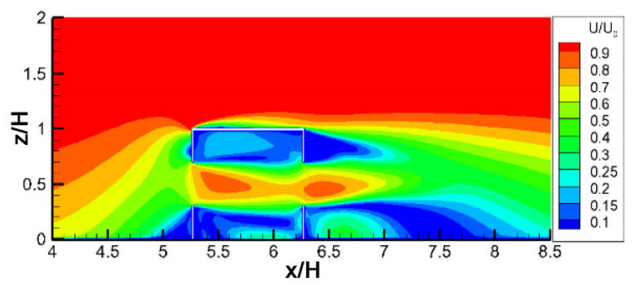

(h) $R e_{\mathrm{w}}=19092$

Fig. 10 Contours of dimensionless velocity $\left(U / U_{0}\right)$ against increasing $R e_{\mathrm{w}}$ numbers

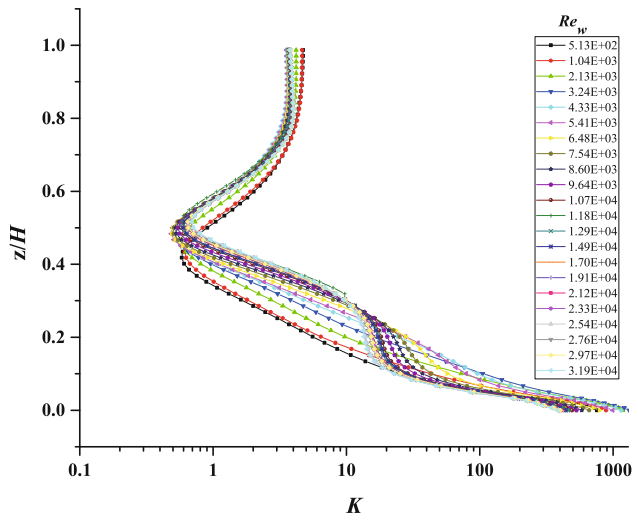

(a) Distributions of dimensionless concentrations along indoor vertical center line with varying $R e_{\mathrm{w}}$



(b) $K \_D R$ vs. $R e_{\mathrm{w}}$

Fig. $11 R e$-independence for indoor pollutant dispersion

concentrations corresponding to two adjacent $R e$ numbers, $R e_{i}$ and $R e_{j}$, respectively. Similar to the previous analysis of $R e$-independence of flow structure, $K \_D R \leq 5 \%$ as the threshold is used to judge the diffusion of pollutants into the $R e$-independence regime. It can be found that when $R e_{\mathrm{w}}<15,000, K \_D R$ will decrease sharply from $22 \%$ down to $5 \%$ with the increasing $R e_{\mathrm{w}}$, indicating this region is strongly $R e$-dependent for the diffusion of pollutants. When 
$R e_{\mathrm{w}}>15,000, K \_D R$ is basically below the standard of $5 \%$, and there are almost no changes with the increase of $R e_{\mathrm{w}}$ that is, the flow enters the $R e$-independence regime for pollutant diffusion. Taking $K \_D R \leq 5 \%$ as the indicator, the suggested values of $R e_{\mathrm{w}, \text { crit }}$ is 15,000 for indoor pollutants diffusion. This critical value is almost the same as the critical value of $R e$-independence for indoor flow structures, indicating that indoor passive diffusion is completely determined by the flow structures and turbulent diffusion.

Figure 12 shows the contours of dimensionless concentration $(K)$ against the increasing $R e_{\mathrm{w}}$ numbers in the indoor vertical center planes. From the figures, it can be noted that the pollutants are mainly concentrated in the lower part below the window after being released from the ground level, then in the upper part above the window because of the vortex structure. Due to the high wind speed and strong ventilation capacity at the window level, the pollutant concentration is the lowest. When $R e_{\mathrm{w}}$ is small

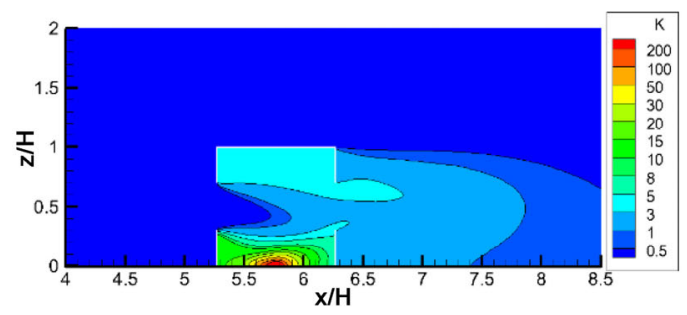

(a) $R e_{\mathrm{w}}=513$

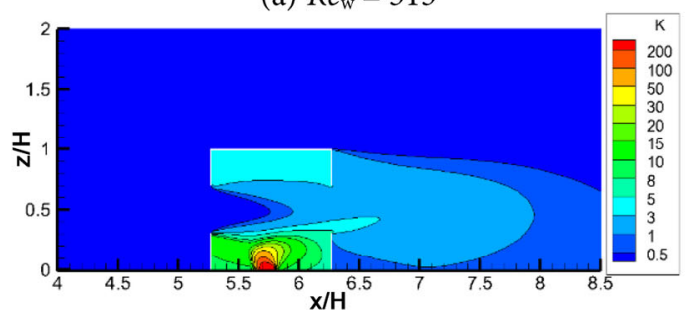

(c) $R e_{\mathrm{w}}=5410$

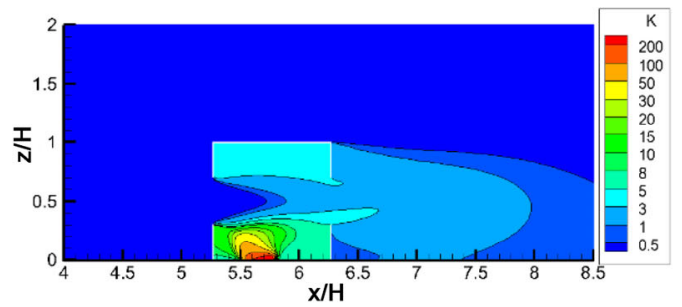

(e) $R e_{\mathrm{w}}=11751$

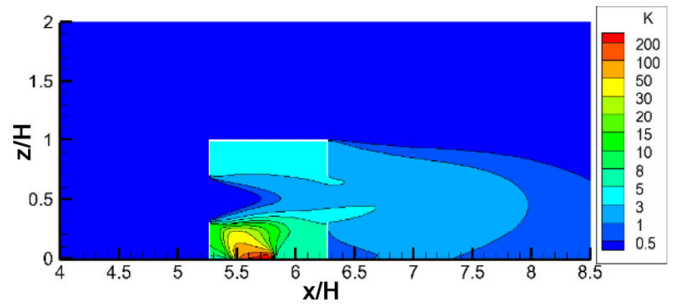

(g) $R e_{\mathrm{w}}=16980$
$\left(R e_{\mathrm{w}}<14,894\right)$, with the increase of $R e_{\mathrm{w}}$, the dimensionless concentration distributions change significantly in the vertical center planes, particularly in the lower part of the window. It can be seen that the high concentration regions gradually moved from the ground center to the left of the lower part. Then with the increase of $R e_{\mathrm{w}}\left(R e_{\mathrm{w}}>9,640\right)$, the dimensionless concentration distribution basically no longer changes in and above the window level, but the concentration distributions in the lower part below the window still change significantly. Until $R e_{\mathrm{w}}>14,894$, the contours of dimensionless concentration in the lower part below the window no longer change with the increase of $R e_{\mathrm{w}}$ and the overall distributions of indoor pollutant concentration can be considered to enter the $R e$-independence regime. Therefore, it is also confirmed from the distributions of the pollutant concentration that the suggested $R e_{\mathrm{w}, \text { crit }}$ for ensuring indoor pollutant diffusion enter the $R e$-independence regime should also be 15,000 .

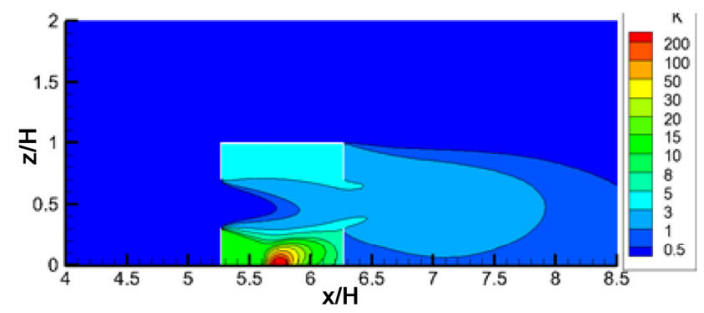

(b) $R e_{\mathrm{w}}=2127$



(d) $R e_{\mathrm{w}}=8599$



(f) $R e_{\mathrm{w}}=14894$

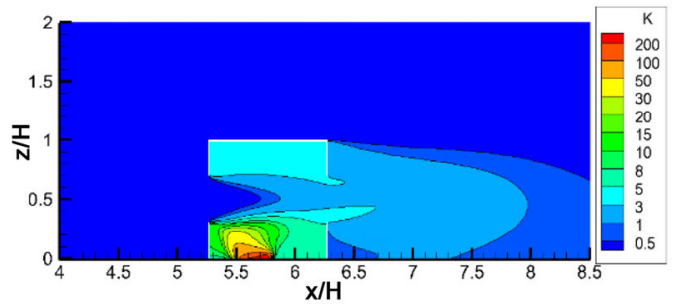

(h) $R e_{\mathrm{w}}=19092$

Fig. 12 Contours of dimensionless concentration $(K)$ against increasing $R e_{\mathrm{w}}$ numbers 


\section{Conclusion}

To ensure the reliability of the data obtained by reducedscale numerical or wind-tunnel models, this study conducted the numerical models validated by wind-tunnel experiments to investigate the issues of $R e$-independence of indoor airflow and pollutant dispersion within an isolated building. The window Reynolds number $\left(R e_{\mathrm{w}}\right)$ was defined as the characteristic $R e$ for indoor airflow and dispersion. The indicators of $R R C$ or $D R\left(K_{-} D R\right)$ less than 5\% were applied to quantitatively determine the critical $R e$ for flow structures and turbulent diffusion, respectively. Some meaningful conclusions can be obtained as follows.

(1) The different Reynolds numbers $\left(R e_{\mathrm{H}}\right.$ and $\left.R e_{\mathrm{w}}\right)$ are required to characterize the flow and dispersion around and within an isolated building. The $R e_{\text {crit }}$ value is position-dependence parameter, it needs different $R e_{\text {crit }}$ to ensure the flow at different locations to enter the Reindependence regime. It is easier for the flow around the building to enter the $R e$-independence regime than the indoor flow structures, resulting from being subject to a relative small viscous force in a semi-infinite space.

(2) The results of quantitatively determining the $R e_{\text {crit }}$ $\left(R e_{\mathrm{H}, \text { rrit }}\right.$ and $\left.R e_{\mathrm{w}, \text { rrit }}\right)$ using the standard of $R R C$ or $D R<5 \%$ shows that $R e_{\text {crit }}$ at the most unfavorable position should be selected as the optimal value to ensure the $R e$-independence of the flows within the whole areas of interest. We found that the wake region on the leeward side of the building is the most unfavorable, thus $R e_{\mathrm{H}, \mathrm{crit}}=27,000$ is recommended for the outdoor flows; while $R e_{\mathrm{w}, \text { crit }}=15,000$ is determined for the indoor flows due to the lower part below the window showing the most unfavorable.

(3) By Comparing the $D R$ vs. $R e_{\mathrm{w}}$ of three window size cases (large $4 W / 3$, medium $W$ and small $2 W / 3$ ), we note that the airflow at the window level is easier to enter the Reindependence regime, and the required Re-dependence will reduce with the increase of window size. However, the suggested $R e_{\mathrm{w}, \text { crit }}(=15,000)$ for indoor airflow and cross ventilation is independence of the window size.

(4) Taking $K_{-} D R \leq 5 \%$ as the indicator, the suggested $R e_{\mathrm{w}, \text { rrit }}$ for ensuring indoor pollutant diffusion enter the $R e$-independence regime should also be 15,000 , indicating that indoor passive diffusion is completely determined by the flow structures and turbulent diffusion. The contours of dimensionless velocity $\left(U / U_{0}\right)$ and concentration $(K)$ against the increasing $R e_{\mathrm{w}}$ further confirmed this critical value.

In this paper, only the effects of cross ventilation on indoor flow and dispersion inside an isolated building are discussed, and the flow regimes in these cases are fully turbulent. In reality, the very small indoor flow flux may be caused by the small vents, single-side ventilation, or the blocking effect of the surrounding buildings. Under these circumstances, the flow structures may be dominated by the viscous forces, therefore, it is worth exploring further whether the concept of Re-independence still applies.

\section{Acknowledgements}

This work was supported by Shanghai Sailing Program (No. 18YF1417600), Scientific and Innovative Action Plan of Shanghai (No. 20dz1204008) and the National Natural Science Foundation of China (No. 51536006). Wind-tunnel experiments were conducted in the EWT Lab of USST.

\section{References}

Ai ZT, Mak CM (2014a). Potential use of reduced-scale models in CFD simulations to save numerical resources: Theoretical analysis and case study of flow around an isolated building. Journal of Wind Engineering and Industrial Aerodynamics, 134: 25-29.

Ai ZT, Mak CM (2014b). Modeling of coupled urban wind flow and indoor air flow on a high-density near-wall mesh: Sensitivity analyses and case study for single-sided ventilation. Environmental Modelling \& Software, 60: 57-68.

Calautit JK, Hughes BR (2016). A passive cooling wind catcher with heat pipe technology: CFD, wind tunnel and field-test analysis. Applied Energy, 162: 460-471.

Castro IP, Robins AG (1977). The flow around a surface-mounted cube in uniform and turbulent streams. Journal of Fluid Mechanics, 79: 307-335.

Cherry NJ, Hillier R, Latour MEMP (1984). Unsteady measurements in a separated and reattaching flow. Journal of Fluid Mechanics, 144: $13-46$.

Chow WK, Gao Y, Wang JL, et al. (2014). Effects of wind, buoyancy and thermal expansion on a room fire with natural ventilation. Building and Environment, 82: 420-430.

Cui P-Y, Li Z, Tao W-Q (2014). Investigation of Re-independence of turbulent flow and pollutant dispersion in urban street canyon using numerical wind tunnel (NWT) models. International Journal of Heat and Mass Transfer, 79: 176-188.

Cui P-Y, Li Z, Tao W-Q (2017). Numerical investigations on Reindependence for the turbulent flow and pollutant dispersion under the urban boundary layer with some experimental validations. International Journal of Heat and Mass Transfer, 106: 422-436.

Dai Y, Mak CM, Ai Z (2019). Flow and dispersion in coupled outdoor and indoor environments: Issue of Reynolds number independence. Building and Environment, 150: 119-134.

Djilali N, Gartshore IS (1991). Turbulent flow around a bluff rectangular plate. Part I: experimental investigation. Journal of Fluids Engineering, 113: 51-59.

Dong L, Zhang X, Xiao Y, et al. (2019). Investigation on Reindependence of air flow and pollutant concentration field in the basement space of an underground sewage treatment plant. Building and Environment, 163: 106327. 
Franke J, Hellsten A, Schlünzen H, et al. (2007). Best Practice Guideline for the CFD Simulation of Flows in the Urban Environment. Brussels: COST Office.

Gilani S, Montazeri H, Blocken B (2016). CFD simulation of stratified indoor environment in displacement ventilation: Validation and sensitivity analysis. Building and Environment, 95: 299-313.

Gousseau P, Blocken B, Stathopoulos T, et al. (2011). CFD simulation of near-field pollutant dispersion on a high-resolution grid: $\mathrm{A}$ case study by LES and RANS for a building group in downtown Montreal. Atmospheric Environment, 45: 428-438.

Gromke C, Buccolieri R, di Sabatino S, et al. (2008). Dispersion study in a street canyon with tree planting by means of wind tunnel and numerical investigations-Evaluation of CFD data with experimental data. Atmospheric Environment, 42: 8640-8650.

Gromke C (2011). A vegetation modeling concept for Building and Environmental Aerodynamics wind tunnel tests and its application in pollutant dispersion studies. Environmental Pollution, 159: 2094-2099.

Gromke C, Ruck B (2012). Pollutant concentrations in street canyons of different aspect ratio with avenues of trees for various wind directions. Boundary-Layer Meteorology, 144: 41-64.

Gupta A, Stathopoulos T, Saathoff P (2012). Wind tunnel investigation of the downwash effect of a rooftop structure on plume dispersion. Atmospheric Environment, 46: 496-507.

Huang Y-d, Li M-z, Ren S-q, et al. (2019). Impacts of tree-planting pattern and trunk height on the airflow and pollutant dispersion inside a street canyon. Building and Environment, 165: 106385.

Ikegaya N, Hasegawa S, Hagishima A (2019). Time-resolved particle image velocimetry for cross-ventilation flow of generic block sheltered by urban-like block arrays. Building and Environment, 147: 132-145.

Jayaweera M, Perera H, Gunawardana B, et al. (2020). Transmission of COVID-19 virus by droplets and aerosols: A critical review on the unresolved dichotomy. Environmental Research, 188: 109819.

Jiru TE, Bitsuamlak GT (2010). Application of CFD in modelling wind-induced natural ventilation of buildings-A review. International Journal of Ventilation, 9: 131-147.

Jones AP (1999). Indoor air quality and health. Atmospheric Environment, 33: 4535-4564.

Kosutova K, van Hooff T, Vanderwel C, et al. (2019). Crossventilation in a generic isolated building equipped with louvers: Wind-tunnel experiments and CFD simulations. Building and Environment, 154: 263-280.

Launder BE, Spalding DB (1974). The numerical computation of turbulent flows. Computer Methods in Applied Mechanics and Engineering, 3: 269-289.

Lim HC, Castro IP, Hoxey RP (2007). Bluff bodies in deep turbulent boundary layers: Reynolds-number issues. Journal of Fluid Mechanics, 571: 97-118.

Meroney RN (1987). Guidelines for fluid modeling of dense gas cloud dispersion. Journal of Hazardous Materials, 17: 23-46.

Mochida A, Murakami S, Kato S (1994). The similarity requirements for wind tunnel model studies of gas diffusion. Wind Engineers, JAWE, 1994(59): 23-28.
Ohba M (1989). Experimental studies for effects of separated flow on gaseous diffusion around two model buildings: Effects of flow conditions of oncoming flow. Journal of Architecture Planning and Environmental Engineering (Transactions of AIJ), 406: 21-30. (in Japanese)

Omrani S, Garcia-Hansen V, Capra BR, et al. (2017). Effect of natural ventilation mode on thermal comfort and ventilation performance: Full-scale measurement. Energy and Buildings, 156: 1-16.

Park J, Sun X, Choi JI, et al. (2017). Effect of wind and buoyancy interaction on single-sided ventilation in a building. Journal of Wind Engineering and Industrial Aerodynamics, 171: 380-389.

Patankar SV (1980). Numerical Heat Transfer and Fluid Flow. New York: McGraw-Hill.

Ramponi R, Blocken B (2012). CFD simulation of cross-ventilation for a generic isolated building: Impact of computational parameters. Building and Environment, 53: 34-48.

Perén JI, van Hooff T, Leite BCC, et al. (2015). CFD analysis of cross-ventilation of a generic isolated building with asymmetric opening positions: Impact of roof angle and opening location. Building and Environment, 85: 263-276.

Robinson J, Nelson WC (1995). National Human Activity Pattern Survey Data Base. Research Triangle Park, NC, USA: United States Environmental Protection Agency.

Saathof PJ, Stathopoulos T, Dobrescu M (1995). Effects of model scale in estimating pollutant dispersion near buildings. Journal of Wind Engineering and Industrial Aerodynamics, 54-55: 549-559.

Saathoff P, Gupta A, Stathopoulos T, et al. (2009). Contamination of fresh air intakes due to downwash from a rooftop structure. Journal of the Air \& Waste Management Association, 59: 343-353.

Shirzadi M, Mirzaei PA, Naghashzadegan M, et al. (2018). Modelling enhancement of cross-ventilation in sheltered buildings using stochastic optimization. International Journal of Heat and Mass Transfer, 118: 758-772.

Shirzadi M, Tominaga Y, Mirzaei PA (2020). Experimental and steady-RANS CFD modelling of cross-ventilation in moderatelydense urban areas. Sustainable Cities and Society, 52: 101849.

Shu C, Wang LL, Mortezazadeh M (2020). Dimensional analysis of Reynolds independence and regional critical Reynolds numbers for urban aerodynamics. Journal of Wind Engineering and Industrial Aerodynamics, 203: 104232.

Snyder WH (1972). Similarity criteria for the application of fluid models to the study of air pollution meteorology. BoundaryLayer Meteorology, 3: 113-134.

Snyder WH (1981). Guideline for Fluid Modeling of Atmospheric Diffusion. Research Triangle Park, NC, USA: United States Environmental Protection Agency.

Tao W-Q (2001). Numerical Heat Transfer. Xi'an, China: Xi'an Jiaotong University Press. (in Chinese)

Tominaga Y, Mochida A, Yoshie R, et al. (2008). AIJ guidelines for practical applications of CFD to pedestrian wind environment around buildings. Journal of Wind Engineering and Industrial Aerodynamics, 96: 1749-1761.

Tominaga Y, Stathopoulos T (2013). CFD simulation of near-field pollutant dispersion in the urban environment: a review of current modeling techniques. Atmospheric Environment, 79: 716-730. 
Townsend AA (1956). The Structure of Turbulent Shear Flow. Cambridge, UK: Cambridge University Press.

Uehara K, Wakamatsu S, Ooka R (2003). Studies on critical Reynolds number indices for wind-tunnel experiments on flow within urban areas. Boundary-Layer Meteorology, 107: 353-370.

van Hooff T, Blocken B (2010a). Coupled urban wind flow and indoor natural ventilation modelling on a high-resolution grid: a case study for the Amsterdam ArenA stadium. Environmental Modelling \& Software, 25: 51-65.

van Hooff T, Blocken B (2010b). On the effect of wind direction and urban surroundings on natural ventilation of a large semienclosed stadium. Computers \& Fluids, 39: 1146-1155. van Hooff T, Blocken B, Tominaga Y (2017). On the accuracy of CFD simulations of cross-ventilation flows for a generic isolated building: Comparison of RANS, LES and experiments. Building and Environment, 114: 148-165.

Wilson N, Corbett S, Tovey E (2020). Airborne transmission of COVID-19. BMJ, 370: $\mathrm{m} 3206$.

Yakhot V, Orszag SA (1986). Renormalization group analysis of turbulence. I. Basic theory. Journal of Scientific Computing, 1: 3-51.

Yee E, Gailis RM, Hill A, et al. (2006). Comparison of wind-tunnel and water-channel simulations of plume dispersion through a large array of obstacles with a scaled field experiment. BoundaryLayer Meteorology, 121: 389-432. 